\title{
THE IMPACT OF EVOLVING INFRARED SPECTRAL ENERGY DISTRIBUTIONS OF GALAXIES ON STAR FORMATION RATE ESTIMATES
}

\author{
R. Nordon ${ }^{1}$, D. Lutz ${ }^{1}$, R. Genzel ${ }^{1}$, S. Berta ${ }^{1}$, S. Wuyts ${ }^{1}$, B. Magnelli ${ }^{1}$, B. Altieri ${ }^{2}$, P. Andreani ${ }^{3}$, H. Aussel ${ }^{4}$, \\ A. Bongiovanni ${ }^{5,6}$, J. Cepa ${ }^{5,6}$, A. Cimatti ${ }^{7}$, E. Daddi ${ }^{4}$, D. FAdDA ${ }^{8}$, N. M. Förster Schreiber ${ }^{1}$, G. Lagache ${ }^{9}$, R. Maiolino ${ }^{10}$, \\ A. M. Pérez García ${ }^{5,6}$, A. Poglitsch ${ }^{1}$, P. Popesso ${ }^{1}$, F. Pozzi ${ }^{10}$, G. Rodighiero ${ }^{11}$, D. Rosario ${ }^{1}$, A. Saintonge ${ }^{1}$,

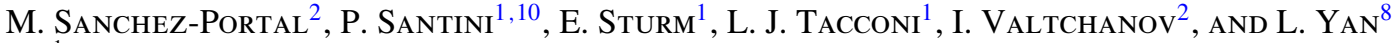 \\ ${ }^{1}$ Max-Planck-Institut für extraterrestrische Physik, Postfach 1312, 85741 Garching, Germany; nordon@mpe.mpg.de \\ ${ }^{2}$ Herschel Science Centre, European Space Astronomy Centre, ESA, Villanueva de al Cañada, 28691 Madrid, Spain \\ ${ }^{3}$ ESO, Karl-Schwarzschild-Str. 2, D-85748 Garching, Germany \\ ${ }^{4}$ Laboratoire AIM, CEA/DSM-CNRS-Université Paris Diderot, IRFU/Service d'Astrophysique, Bât.709, CEA-Saclay, 91191 Gif-sur-Yvette Cedex, France \\ ${ }^{5}$ Instituto de Astrofísica de Canarias, 38200 La Laguna, Tenerife, Spain \\ ${ }^{6}$ Departamento de Astrofísica, Universidad de La Laguna, La Laguna, Spain \\ ${ }^{7}$ Dipartimento di Astronomia, Università di Bologna, Via Ranzani 1, 40127 Bologna, Italy \\ ${ }^{8}$ IPAC, California Institute of Technology, Pasadena, CA 91125, USA \\ ${ }^{9}$ Institut d'Astrophysique Spatiale (IAS), Bât 121, Université de Paris XI, 91450 Orsay Cedex, France \\ ${ }^{10}$ INAF-Osservatorio Astronomico di Roma, via di Frascati 33, 00040 Monte Porzio Catone, Italy \\ ${ }^{11}$ Department of Astronomy, University of Padova, Vicolo dell'Osservatorio 3, 35122 Padova, Italy \\ Received 2011 June 5; accepted 2011 November 7; published 2012 January 17
}

\begin{abstract}
We combine Herschel-Photodetector Array Camera and Spectrometer (PACS) data from the PACS Evolutionary Probe (PEP) program with Spitzer $24 \mu \mathrm{m}$ and $16 \mu \mathrm{m}$ photometry and ultra deep Infrared Spectrograph (IRS) midinfrared spectra to measure the mid- to far-infrared spectral energy distribution (SED) of $0.7<z<2.5$ normal star-forming galaxies (SFGs) around the main sequence (the redshift-dependent relation of star formation rate (SFR) and stellar mass). Our very deep data confirm from individual far-infrared detections that $z \sim 2$ SFRs are overestimated if based on $24 \mu \mathrm{m}$ fluxes and SED templates that are calibrated via local trends with luminosity. Galaxies with similar ratios of rest-frame $v L_{v}(8)$ to $8-1000 \mu \mathrm{m}$ infrared luminosity (LIR) tend to lie along lines of constant offset from the main sequence. We explore the relation between SED shape and offset in specific star formation rate (SSFR) from the redshift-dependent main sequence. Main-sequence galaxies tend to have a similar $v L_{v}(8) /$ LIR regardless of LIR and redshift, up to $z \sim 2.5$, and $v L_{v}(8) /$ LIR decreases with increasing offset above the main sequence in a consistent way at the studied redshifts. We provide a redshift-independent calibration of SED templates in the range of 8-60 $\mu \mathrm{m}$ as a function of $\Delta \log (\mathrm{SSFR})$ offset from the main sequence. Redshift dependency enters only through the evolution of the main sequence with time. Ultra deep IRS spectra match these SED trends well and verify that they are mostly due to a change in ratio of polycyclic aromatic hydrocarbon (PAH) to LIR rather than continua of hidden active galactic nuclei (AGNs). Alternatively, we discuss the dependence of $v L_{v}(8) / \mathrm{LIR}$ on LIR. The same $v L_{v}(8) /$ LIR is reached at increasingly higher LIR at higher redshift, with shifts relative to local by 0.5 and $0.8 \mathrm{dex}$ in $\log (\mathrm{LIR})$ at redshifts $z \sim 1$ and $z \sim 2$. Corresponding SED template calibrations are provided for use if no stellar masses are on hand. For most of those $z \sim 2$ SFGs that also host an AGN, the mid-infrared is dominated by the star-forming component.
\end{abstract}

Key words: cosmology: observations - galaxies: evolution - galaxies: fundamental parameters - galaxies: starburst - infrared: galaxies

Online-only material: color figures

\section{INTRODUCTION}

In any study of galaxy evolution, the star formation rate (SFR) is a key parameter. SFRs can be estimated in various ways from different wavelengths, but for all reasonably massive and dusty star-forming galaxies (SFGs), the far-infrared emission will dominate the emitted power and provide a reliable SFR estimator. In SFGs most of the light which is emitted from young stars is absorbed by dust and re-emitted in the farinfrared, peaking around $60-100 \mu \mathrm{m}$ rest wavelength. Thus, a measurement or an estimate of the total infrared luminosity (LIR, integrated between rest-frame 8 and $1000 \mu \mathrm{m}$ ) of a galaxy is required in order to account for the reprocessed fraction of the light.

The inaccessibility of the far-infrared to ground-based instruments and the technological challenges of far-infrared space missions have, until recently, left this critical part of the spectrum largely unexplored in high-redshift galaxies. Other wavelengths have been used instead to estimate LIR, and the mid-infrared, in particular observed by the Spitzer Space Telescope, has been important in detecting high-redshift, star-forming, and dustobscured galaxies. The mid-infrared itself however accounts for only a small fraction of the LIR, and at increasing redshifts, the mid-infrared band covers even shorter rest-frame wavelengths, on the edge of the relevant $8-1000 \mu \mathrm{m}$ range. The derivation of LIR from mid-infrared observations requires large extrapolations that carry significant uncertainties. These extrapolations are typically based on families of spectral energy distributions (SEDs) that are parameterized as a function of LIR and calibrated to match the trends observed in the local universe, in particular the library of Chary \& Elbaz (2001, CE01 in the following).

Indications that local infrared SEDs may not hold emerged with the first more detailed high- $z$ studies. Luminous 
submillimeter galaxies (SMGs) were inferred to have lower far-infrared dust temperatures (e.g., Chapman et al. 2005; Pope et al. 2006), and larger polycyclic aromatic hydrocarbon (PAH) equivalent width and ratio to LIR (e.g., Lutz et al. 2005; Valiante et al. 2007; Menéndez-Delmestre et al. 2007; Pope et al. 2008; Murphy et al. 2009), when compared to local sources of equivalent LIR. Strong PAH emission was also found in some luminous "dust-obscured galaxies" (DOGs) selected by high $24 \mu \mathrm{m}$ to optical flux ratios (e.g., Weedman et al. 2006; Sajina et al. 2007; Murphy et al. 2009). Both types of selections induce SED bias and studies typically referred to luminous LIR $\gtrsim 10^{12.5} L_{\odot}$ sources. These limitations apply much less to studies of a small number of lensed galaxies (Desai et al. 2006; Rigby et al. 2008; Siana et al. 2008, 2009; Fadely et al. 2010), again typically indicating changing SEDs and stronger PAH than in local galaxies of similar LIR. Using 70 and $160 \mu \mathrm{m}$ stacking of $24 \mu \mathrm{m}$ sources (Papovich et al. 2007), radio stacking of BzK galaxies (Daddi et al. 2007b), and an SED study including Infrared Spectrograph (IRS) spectra of a sample of SMGs, DOGs, and active galactic nucleus (AGN) hosts (Murphy et al. 2009), evidence was then found that extrapolations from Spitzer $24 \mu \mathrm{m}$ mid-infrared photometry tend to overestimate LIR for $z \sim 2$ galaxies by factors of 2-10. This concerns a key epoch of galaxy evolution, during which both cosmic star formation and AGN activity peak, and which is thus at the focus of many current observational and theoretical studies. Whether this relative "mid-infrared excess" is due to continuum emission from dust heated by obscured AGNs that boost mid-infrared fluxes (Daddi et al. 2007a), or due to a mismatch between the real SEDs at high- $z$ and the locally calibrated templates that are used in converting the mid-infrared flux to LIR, remained unclear. Stacking of X-ray data revealed a large fraction of obscured AGNs in "mid-infrared excess" sources (Daddi et al. 2007a), but then evidence from IRS spectroscopy pointed toward strong emission from PAH not fully accounted for by the templates (Murphy et al. 2009; Fadda et al. 2010).

At $z>1.5$, the strong rest-frame $8 \mu \mathrm{m}$ PAH complex is redshifted into the $24 \mu \mathrm{m}$ filter, which raised the suspicion that the local template SEDs have too low ratios of PAH to far-infrared emission, which in turn lead to inaccurate extrapolations. This ratio varies significantly among local galaxies. Extrapolations from $8 \mu \mathrm{m}$ flux to LIR thus critically depend on selecting the correct SED template.

The $8 \mu \mathrm{m}$ PAH complex includes several broad emission bands. Its flux relative to the far-infrared depends strongly on the local interstellar medium (ISM) conditions (Laurent et al. 2000; Sales et al. 2010). In the local SED family of CE01, the ratio of $8 \mu \mathrm{m}$ PAH to LIR is fairly constant at LIR $\lesssim 10^{10} L_{\odot}$ where it is based mostly on normal star-forming disks. At higher LIR where the ratio of the $8 \mu \mathrm{m}$ luminosity to LIR decreases, it is based on low-redshift luminous infrared galaxies (LIRGs, LIR > $10^{11} L_{\odot}$ ) and ultraluminous infrared galaxies (ULIRGs, LIR > $10^{12} L_{\odot}$ ). Local (U)LIRGs have distinct properties that make them differ from an aggregation of numerous disk star-forming regions. They are typically interacting or merging systems (Sanders \& Mirabel 1996) with their luminous star formation concentrated in a small region of size a few $100 \mathrm{pc}$ (Condon et al. 1991). They show high "star formation efficiencies" $\mathrm{SFR} / M_{\mathrm{Gas}}$, lower CO luminosity to gas mass conversion factor (Solomon et al. 1997; Downes \& Solomon 1998), and a "deficit" of [C II] 158 compared to LIR (e.g., Malhotra et al. 1997; Luhman et al. 2003; Graciá-Carpio et al. 2011). All these properties reflect a compact structure and intense radiation fields. The intense radiation fields naturally lead to lower $\mathrm{PAH} / \mathrm{LIR}$ and a warmer far-infrared dust peak (e.g., Dale et al. 2001), placing ULIRGs at the high temperature end of the local compactness-temperature relation (Chanial et al. 2007).

All these well-established properties and connotations of the local ULIRGs, beyond the basic definition of the class by LIR, do not necessarily apply for equally luminous $z \sim 2$ galaxies. High-redshift galaxies with ULIRG-like luminosities are more gas-rich (e.g., Tacconi et al. 2010), can be disk-like galaxies without indication of recent mergers (e.g., Shapiro et al. 2008; Förster Schreiber et al. 2009), and are not as compact as the local ULIRGs (Bouché et al. 2007; Kriek et al. 2009; Förster Schreiber et al. 2011). Along with this come arguments for more normal star formation efficiencies (Genzel et al. 2010; Daddi et al. 2010b) and less of a [C II] deficit (e.g., Maiolino et al. 2009; Graciá-Carpio et al. 2011). Changes in star formation efficiencies and [C II] deficit reflect changing local ISM conditions in high-redshift galaxies compared to local ones of the same luminosity, and challenge the association of the absolute LIR with a single template SED shape.

In recent years, a correlation has been established between the SFR and stellar mass of SFGs (Noeske et al. 2007; Daddi et al. 2007b; Elbaz et al. 2007). This is now commonly referred to as the "main sequence" of SFGs, from which passive early type galaxies and local ULIRGs are outliers. The absolute scaling and possibly the slope of the specific star formation rate (SSFR, i.e., SFR per unit stellar mass) main sequence evolve with redshift. The SSFR significantly increases with redshift, scaling as $(1+z)^{n}$, where $2.2<n<4$ (Daddi et al. 2007b; Erb et al. 2006; Damen et al. 2009; Dunne et al. 2009; Pannella et al. 2009; Bouché et al. 2010; Rodighiero et al. 2010; Karim et al. 2011). There is an ongoing debate on the slope $\beta$ of the SSFR $\propto M_{*}^{\beta}$ main sequence of SFGs. At $z \sim 1-2$, much of the variation that is found may be related to how specifically SFGs are separated from passive ones. BzK color selections seem to produce flatter slopes near $\beta=-0.1$ (Daddi et al. 2007b; Pannella et al. 2009) compared to $\beta=-0.4 \ldots-0.5$ when isolating SFGs in other ways from original mass selections (Rodighiero et al. 2010; Karim et al. 2011); see also the direct comparison in Karim et al. (2011).

One of the implications of this fairly tight correlation between SFR and mass is that star formation in these objects must be relatively continuous. This raises yet another reason to question the applicability of mid- to far-infrared flux ratios that were calibrated on local ULIRGs with strongly peaked star formation histories. The possibility arises that SSFR or its offset from the main sequence may more generally define the SED shape, rather than LIR alone.

With the advent of the Herschel ${ }^{12}$ space telescope (Pilbratt et al. 2010) and its Photodetector Array Camera and Spectrometer (PACS) instrument (Poglitsch et al. 2010), main-sequence SFGs at redshifts of $z \sim 1-2$ can now be detected directly in the 100 and $160 \mu \mathrm{m}$ bands, reaching objects with SFRs as low as $10 \mathrm{~s}$ to a few $100 \mathrm{~s} M_{\odot} \mathrm{yr}^{-1}$ and typical masses above $10^{10} M_{\odot}$. This is due to the Herschel surveys reaching down to very faint fluxes of order $1 \mathrm{mJy}$ (Lutz et al. 2011; Berta et al. 2011). New Herschel-PACS results confirmed and extended the finding that extrapolations from observed $24 \mu \mathrm{m}$ wavelengths for $z>1.5$ galaxies tend to overestimate LIR by factors of four to seven (Nordon et al. 2010; Elbaz et al. 2010). This is in contrast to

\footnotetext{
12 Herschel is an ESA space observatory with science instruments provided by European-led Principal Investigator consortia and with important participation from NASA.
} 
Table 1

PACS Photometry Depths in the Fields Used in this Study

\begin{tabular}{lccc}
\hline \hline Field & \multicolumn{3}{c}{$3 \sigma$ Flux Limits (mJy) } \\
\cline { 2 - 4 } & $70 \mu \mathrm{m}$ & $100 \mu \mathrm{m}$ & $160 \mu \mathrm{m}$ \\
\hline GOODS-N & $\ldots$ & 3.0 & 5.2 \\
GOODS-S & 1.2 & 1.1 & 2.0 \\
\hline
\end{tabular}

lower redshifts where the extrapolations are reasonably accurate (Elbaz et al. 2010). Using data from the GOODS-Herschel program, Elbaz et al. (2011) study the ratio of total IR luminosity to rest-frame $8 \mu \mathrm{m}$ luminosity, which they find to be universal for most SFGs, defining an IR main sequence in SFR versus mass. The outliers (enhanced SFR) to this relation are found to be starbursting galaxies with high projected star formation surface densities. We compare our work to their results in the discussion.

In this paper, we study the relations between the mass, SFR (also LIR), and mid- to far-infrared SED properties of distant SFGs. We focus on two redshift bins: $1.5<z<2.5$ and $0.7<$ $z<1.3$ where the $8 \mu \mathrm{m}$ PAH emission complex is covered by the Spitzer Multiband Imaging Photometer for Spitzer (MIPS) $24 \mu \mathrm{m}$ and IRS blue peak-up $(16 \mu \mathrm{m})$ filters (MIPS24 and IRS16 hereafter), respectively. Herschel-PACS provides the crucial LIR measurements and samples the far-infrared SED shape. We find that galaxies with constant $\nu L_{\nu}(8 \mu \mathrm{m})$ (where $v L_{v}=4 \pi D_{\text {lumin }}^{2} \nu F_{v}$ ) to LIR emission ratios tend to lie in lines parallel to the main sequence (Section 3.1). This motivates us to study the SED properties of galaxies as a function of their distance from the redshift-dependent main sequence, in addition to the traditional view as a function of IR luminosity. We adopt the redshift-dependent main sequence from Rodighiero et al. (2010), which is based on Herschel-PACS data closely related to our sample.

In Sections 2 and 3, we describe our sample, reconfirm from our deeper data the mid-infrared excess overprediction of $z \sim 2$ SFRs when extrapolated from $24 \mu \mathrm{m}$, and motivate the discussion of SEDs as a function of offset from the main sequence. In Section 4, we describe the mean SED redshift scan fitting method. In Section 5, we study the mid- to farinfrared SEDs as a function of distance from the star-forming main sequence, demonstrating that this provides a calibration without explicit redshift dependence. We then, in Section 6, compare IRS spectra with the modified templates. This also demonstrates that the change in mid-infrared SEDs is indeed due to scaling of the relative PAH strength and not due to continuum emission from hot dust. In Section 7, we alternatively present the SEDs in the more traditional way as a function of luminosity and derive a redshift-dependent calibration of $\nu L_{v}(8 \mu \mathrm{m})$ as a function of LIR. This calibration allows the use of rest-frame $8 \mu \mathrm{m}$ photometry as an SFR indicator without added knowledge of the mass. In Section 8, we discuss mid- to far-infrared SEDs of X-ray AGN hosts in light of the findings for inactive galaxies.

We adopt a $\left(\Omega_{m}, \Omega_{\Lambda}, H_{0}\right)=\left(0.3,0.7,70 \mathrm{~km} \mathrm{~s}^{-1} \mathrm{Mpc}^{-1}\right)$ cosmology throughout this paper. A Chabrier (2003) initial mass function (IMF) is always assumed. Unless specified otherwise, we refer to the "mid-infrared" as the rest-frame wavelengths corresponding to the MIPS $24 \mu \mathrm{m}$ and IRS $16 \mu \mathrm{m}$ blue peak-up filters and to the "far-infrared" as the wavelengths covered by the PACS $70-160 \mu \mathrm{m}$ filters. For SFGs at redshift $0.7<z<$ 2.5 , these, respectively, correspond to a region dominated by $\mathrm{PAH}$ emission features and by the rest-frame far-infrared peak and its short wavelength slope.
Table 2

Number and Classification of Sources in the $z \sim 1$ and $z \sim 2$ Samples

\begin{tabular}{lccc}
\hline \hline Redshift & SFG & $\begin{array}{c}\text { X-ray } \\
\text { Detected }^{\text {a }}\end{array}$ & $\begin{array}{c}\text { Power } \\
\text { Law }^{\text {a }}\end{array}$ \\
\hline $0.7<z<1.3$ & $148 / 79$ & $21 / 26$ & $1 / 2$ \\
$1.5<z<2.5$ & $106 / 22$ & $16 / 10$ & $13 / 5$ \\
\hline
\end{tabular}

Notes. The two numbers in each cell refer to the GOODS-S/GOODS-N fields. a The X-ray detection and IRAC-bands power-law tags are not mutually exclusive.

\section{DATA AND SAMPLES}

Our far-infrared data are based on Herschel-PACS observations in the GOODS fields. These were obtained as part of the guaranteed-time PACS Evolutionary Probe $\left(\mathrm{PEP}^{13}\right)$ project. For details on the observation field layout and on data reduction, we refer to Lutz et al. (2011). The limiting fluxes are listed in Table 1; for a distribution of source LIR versus redshift, see Figure 12 of Lutz et al. (2011). The PACS 160, 100, and $70 \mu \mathrm{m}$ fluxes were extracted using sources from a Spitzer MIPS $24 \mu \mathrm{m}$ catalog as priors, following the method described in Magnelli et al. (2009). The $24 \mu \mathrm{m}$ images are very deep $(\approx 30 \mu \mathrm{Jy}, 5 \sigma)$ and do not limit the completeness of the prior extraction, because only a very small fraction $(\lesssim 1 \%)$ of PACS detections will be without a $24 \mu \mathrm{m}$ counterpart at these depths (see Lutz et al. 2011; Magdis et al. 2011 for a more in-depth analysis). The prior extraction method naturally matches a $24 \mu \mathrm{m}$ source to a PACS source.

For our sample selection we require a redshift (either photometric or spectroscopic) and a $160 \mu \mathrm{m}$ detection, implying also a $24 \mu \mathrm{m}$ detection. The total number of sources and their breakdown by redshift bins and SFG/AGN categories are summarized in Table 2 . To complete the coverage of the mid-infrared at $z \sim 1$, we also make use of Spitzer-IRS blue peak-up $16 \mu \mathrm{m}$ catalogs by Teplitz et al. (2011). The limiting depths $(3 \sigma)$ in GOODS-N and GOODS-S are 40 and $65 \mu \mathrm{Jy}$, which result in a high detection fraction of PACS sources. In the area covered by the $16 \mu \mathrm{m}$ photometry, at redshifts $0.7<z<1.3,92 \%$ and $77 \%$ of the PACS sources in GOODS-N and GOODS-S fields, respectively, have $16 \mu \mathrm{m}$ counterparts. Of the PACS sources in these respective fields, $12 \%$ and $6 \%$ are outside the $16 \mu \mathrm{m}$ coverage.

Photometric redshifts in GOODS-N were derived using the code EAZY (Brammer et al. 2008) applied on a pointspread function (PSF) matched multi-wavelength catalog in UV (Galaxy Evolution Explorer; Barger et al. 2008; Martin et al. 2005), $U$ band (KPNO 4 m/MOSAIC; Barger et al. 2008; Capak et al. 2004), optical (Advanced Camera for Surveys, Hubble Space Telescope bviz; Giavalisco et al. 2004), near-IR (FLAMINGOS, $J H K_{s}{ }^{14}$ ) and $K_{s}$ band from Subaru/WIRCam (Wang et al. 2010), and mid-infrared (Spitzer Infrared Array Camera (IRAC) and MIPS; M. Dickinson et al. 2012, in preparation). Spectroscopic redshifts in GOODS-N were adopted from Barger et al. (2008). In comparison to the $z_{\text {spec }}$, the $z_{\text {phot }}$ in GOODS-N have a good accuracy with the following statistics for $\Delta_{z}=\left(z_{\text {phot }}-z_{\text {spec }}\right) /\left(1+z_{\text {spec }}\right)$ : median of -0.008 and median absolute deviation (MAD) of 0.033 . The rate of $z_{\text {phot }}$ critical failures, defined here as: $\Delta_{z}>0.5 \Delta \lambda_{\text {MIPS2 }} / 24$, i.e., a redshift deviation that produces a corresponding wavelength deviation

\footnotetext{
13 http://www.mpe.mpg.de/ir/Research/PEP/

14 Kindly reduced by Kyoungsoo Lee.
} 
Table 3

Numbers and Luminosity Ranges of the Full SFG Samples and the SFG Samples Observed with IRS

\begin{tabular}{lcrccc}
\hline \hline Redshifts & Sample & $N$ & \multicolumn{3}{c}{$\log \left(\mathrm{LIR}_{8}-1000 \mu \mathrm{m} / L_{\odot}\right)$} \\
\cline { 3 - 6 } & & & Min & Median & Max \\
\hline $0.7<z<1.3$ & SFG & 227 & 10.50 & 11.36 & 12.2 \\
& SFG-IRS & 9 & 11.15 & 11.59 & 11.92 \\
\hline $1.5<z<2.5$ & SFG & 128 & 11.34 & 12.0 & 12.87 \\
& SFG-IRS & 16 & 11.63 & 11.97 & 12.72 \\
\hline
\end{tabular}

Note. AGNs are excluded.

greater than the half-width of the $24 \mu \mathrm{m}$ filter, is $7 \%$. Spectroscopic redshifts in GOODS-S were adopted from Balestra et al. (2010) matched to the multi-wavelength (bvizJHK, IRAC, MIPS $24 \mu \mathrm{m}$ ) MUSIC catalog (Santini et al. 2009) which also includes photometric redshifts for the remaining sources. The corresponding $z_{\text {phot }}$ quality statistics for $\Delta_{z}$ are median -0.0025 , MAD 0.038 , critical failures $10 \%$. In total, $88 \%$ of the $z \sim 1$ and $35 \%$ of the $z \sim 2$ samples have spectroscopic redshifts.

Masses were calculated from the above-mentioned multiwavelength catalogs of both fields using the method described in Fontana et al. (2004), with adjustments as described in Santini et al. (2009); see also Fontana et al. (2006) and Grazian et al. (2006). To ensure good mass estimates when discussing trends with SSFR, we require for those samples a detection in all of the 3.6-5.8 $\mu \mathrm{m}$ IRAC bands that cover the SED rest-frame stellar bump (rest-frame $H$ band). Only six $160 \mu \mathrm{m}$ sources in our redshift bins do not match this criterion; these are included in Tables 2 and 3 but not used below when masses are required. We estimate the error on the derived masses by varying the input photometric fluxes according to their respective errors and find that the median error for the galaxies in our sample is 0.2 dex. While this uncertainty is non-negligible on individual objects, it plays a much reduced role in a statistics-driven study such as the one presented here. In particular, the masses are used in measuring the galaxy SSFR and its difference from the main sequence, which has a width of 0.3-0.4 dex in SSFR (e.g., Elbaz et al. 2007).

Sources that have an X-ray counterpart in the Chandra 2 Ms catalogs (Alexander et al. 2003; Luo et al. 2008) have conservatively been flagged as AGNs. At a limit of $\sim 2 \times$

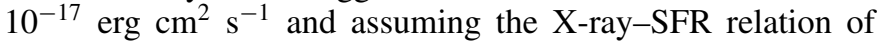
Ranalli et al. (2003), the minimum X-ray luminosity of $z \sim 2$ galaxies that can be detected is too bright for star formation except for extreme SFRs well above those typical for our sample. This is more of a consideration for the $z \sim 1$ sample where the galaxies with the highest SFRs can potentially produce enough X-rays to be detected. When applying the Bauer et al. (2004) classification based on X-ray luminosity and hardness ratio, $18 \%$ of the $z \sim 1$ AGN-flagged sources may actually be X-ray-detected SFGs. Even if $18 \%$ of the AGNs (3\% of the full PACS sample) in the $0.7<z<1.3$ range are star formation dominated in the X-ray, the removal of these sources from the SFGs sample does not have a significant influence. None of the $z \sim 2$ X-ray sources in our sample is suspected to be star formation dominated according to the Bauer et al. (2004) classification, though some have very low X-ray photon counts, which makes such a classification highly uncertain. Alexander et al. (2005) investigated luminous LIR $\sim 10^{13} L_{\odot}$ SMGs in the GOODS-N field. Among 20 SMGs, 15 are X-ray detected with AGN-related X-rays, 3 are not X-ray detected, and only 2
(10\%) are X-ray detected with X-rays reported to be dominated by star formation. This suggests that at $z \sim 2$ the probability to erroneously flag these most luminous SFGs as an AGN, solely based on an X-ray detection in the Chandra 2 Ms catalog is $\lesssim 10 \%$. This probability drops sharply toward lower LIRs typical of our sample. Sources that show a monotonic rise in flux in the Spitzer-IRAC 3.6-8.0 $\mu \mathrm{m}$ bands are flagged as power-law AGNs. Unless explicitly stated otherwise, the X-ray and powerlaw AGNs are excluded from the analysis.

In this study, we use the deep Spitzer-IRS spectra presented by Fadda et al. (2010). This spectroscopic sample includes 22 sources at $1.75<z<2.4$ and 10 at $0.76<z<1.05$ that are in the PEP GOODS-S field and have a $160 \mu \mathrm{m}$ detection. Five of the $z \sim 2$ sources and one of the $z \sim 1$ sources have an associated $\mathrm{X}$-ray source in the Chandra $2 \mathrm{Ms}$ catalog. This sample selects faint $24 \mu \mathrm{m}$ sources $(0.14-0.5 \mathrm{mJy})$ and probes the common rest-frame wavelength of $5-12 \mu \mathrm{m}$. Table 3 summarizes the numbers and luminosity ranges covered by the full SFG samples and the IRS spectroscopy subsamples.

\section{THE MID-INFRARED EXCESS: OVERPREDICTION OF $z \sim 2$ SFRs BY MID-INFRARED EXTRAPOLATION}

Spitzer-based findings of overpredicted SFRs based on $24 \mu \mathrm{m}$ photometry at $1.5<z<2.5$ (the "mid-infrared excess" mentioned in the introduction) were corroborated and extended by direct measurements of the total LIR in the far-infrared in Nordon et al. (2010) and Elbaz et al. (2010). These measurements used GOODS-N imaging with the PACS and SPIRE instruments from the Herschel science demonstration phase. While resolving many individual sources at these redshifts, they still relied on stacking techniques for a large fraction of the sources: $5 \%$ of PACS detections for the $24 \mu \mathrm{m}$-selected sample in Nordon et al. (2010) and could not probe luminosities smaller than $10^{12} L_{\odot}$. Our current GOODS-S data are about a factor of three deeper than the GOODS-N data used before (Table 1). The deeper data significantly increase the $160 \mu \mathrm{m}$ detection fraction of $24 \mu \mathrm{m}$ sources $(30 \%)$, making stacks less necessary. It also allows us to probe down to $\log \left(\mathrm{LIR} / L_{\odot}\right) \approx 11.3$, which we could not effectively reach before.

In Figure 1, using the GOODS-S data, we confirm the results of Nordon et al. (2010) and Elbaz et al. (2010; see also upper left panel of Figure 13 for a different representation). We plot LIR(160), the LIR as measured from PACS $160 \mu \mathrm{m}$, versus LIR(24), the LIR as extrapolated from MIPS $24 \mu \mathrm{m}$ flux using the Chary \& Elbaz (2001) SED library. MIPS sources undetected by PACS are stacked in LIR(24) bins. Black triangles are the simple, number-weighted means of stacks and detections in the LIR(24) range of each stack. All the procedures are similar to those used in Nordon et al. (2010).

The $24 \mu \mathrm{m}$ "excess" is clearly evident in Figure 1. At the high luminosities, the LIR(24) overestimation is by a factor of $\sim 4$ going down to $\sim 2.5$ toward $\log \left(\mathrm{LIR} / L_{\odot}\right) \approx 11$ which we could not probe directly before. In the following sections, we will demonstrate that this apparent excess is entirely a result of using the wrong SED templates in the $24 \mu \mathrm{m}$ flux to LIR conversion.

\subsection{What Determines the Mid- To Far-IR SED?}

Traditionally, the use of mid-infrared photometry as an SFR indicator relied on empirical correlations between the mid-infrared spectral features and the total LIR. Since the mid-infrared itself accounts for only a small fraction of the 


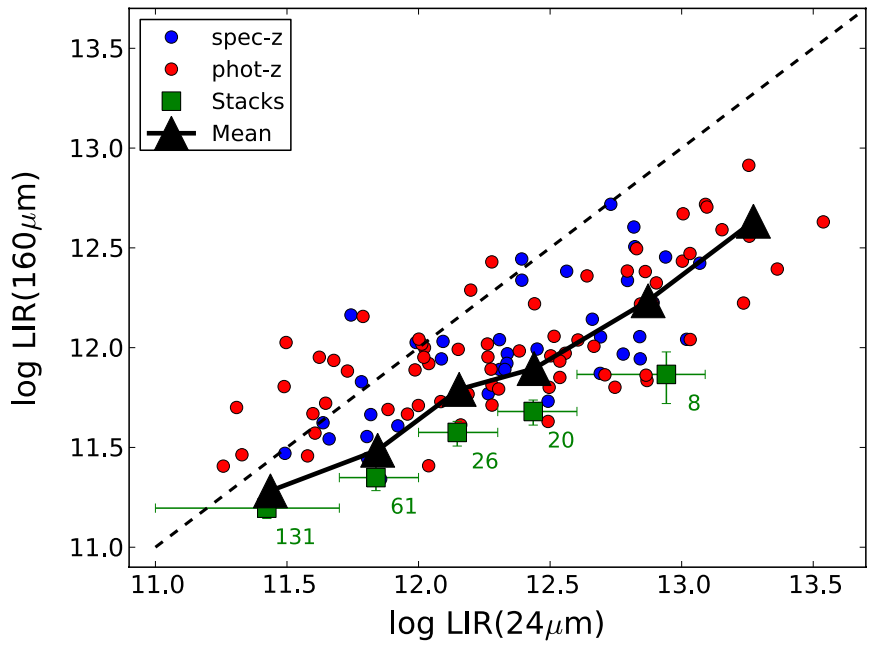

Figure 1. "Mid-infrared excess" of $1.5<z<2.5$ star-forming galaxies in GOODS-S: LIR derived from PACS $160 \mu \mathrm{m}$ vs. LIR derived from MIPS $24 \mu \mathrm{m}$ using the original CE01 templates. Circles indicate individual detections. Squares indicate stacked $24 \mu \mathrm{m}$ sources undetected by PACS, where the number of stacked sources is displayed, the horizontal bars indicate the range of LIR(24) in the stack, and the vertical are the error on the mean LIR(160). The numberweighted mean of stacks and detections is plotted in black triangles and thick black line.

(A color version of this figure is available in the online journal.)

8-1000 $\mu \mathrm{m}$ LIR, such correlations are not trivial. If SED shape were strictly related to LIR and given the main-sequence correlation between stellar mass and LIR, one would expect mass and mid- to far-infrared SED properties to correlate. Dependence of SED on other parameters is expected however, as mentioned in the introduction. As we will demonstrate in this section, there is a dependence of the $v L_{v}(8) / \mathrm{LIR}$ ratio on both mass and LIR, such that galaxies of constant $v L_{v}(8) /$ LIR lie parallel to the main sequence on the SSFR versus mass diagram.

Out of our full sample, we consider in this section only the galaxies with a well-determined stellar mass and a redshift range of $1.8<z<2.3$ (narrower than the full sample). At this redshift range the $7.7 \mu \mathrm{m}$ PAH complex is inside the MIPS24 filter and the $10 \mu \mathrm{m}$ silicate absorption only affects the filter fluxes to a limited degree. Here and consistently throughout the paper, when quoting $v L_{v}(8) /$ LIR we refer to the value that would be measured with a MIPS24 filter for a $z=2.0$ galaxy of the given SED shape. We correct the observed MIPS $24 \mu \mathrm{m}$ $\nu L_{v}(24 / 1+z)$ to $8 \mu \mathrm{m}$ rest-frame fluxes by finding the CE01 template which best fits the $v L_{v}(24 / 1+z) /$ LIR of the filter and the galaxy redshift, then redshifting this template to $z=$ 2 and extracting $v L_{v}(8) /$ LIR for the MIPS24 filter. For the subsample used in this section the extrapolation is over a short rest wavelength interval, and the associated uncertainties are negligible.

Figure 2 plots this subsample on the SSFR versus $M_{*}$ diagram. Red and blue colors indicate galaxies with low $<-0.95$ and high $>-0.95 v L_{v}(8) /$ LIR ratios, which splits the sample roughly in half. While the separation is not clean, it is clear that sources with low $v L_{v}(8) /$ LIR tend to have higher SSFR at any given mass. Probing by eye the somewhat blurred border between the blue and red points, it appears to be sloped rather than at a horizontal line of constant SSFR. Lines of constant LIR appear in this plot at a slope of -1 (gray dotted lines). The blue/red separation is not as steep as these lines of constant LIR. The main sequence from Rodighiero et al. (2010), which is based on Herschel-PACS GOODS-N data, is plotted as a

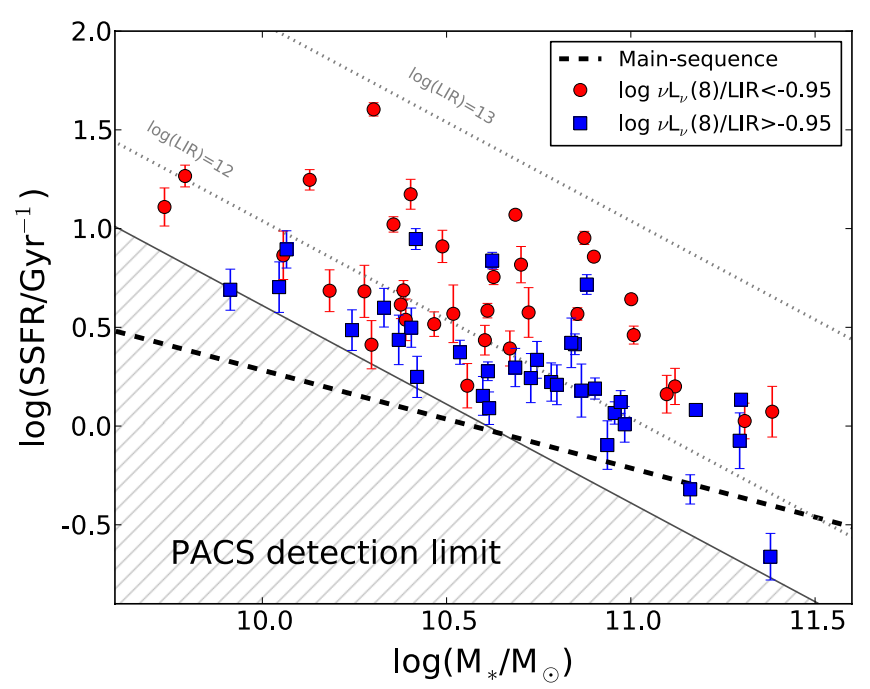

Figure 2. SSFR vs. mass for $1.8<z<2.3$ galaxies. Galaxies with $\log \left(v L_{v}(8) / \mathrm{LIR}\right)<-0.95$ are in red and galaxies with $\log \left(v L_{v}(8) / \mathrm{LIR}\right)>$ -0.95 are in blue. The dashed line is the main sequence of Rodighiero et al. (2010) with a slope of -0.5 . Gray dotted lines with a slope of -1 indicate lines of constant LIR. The gray hatched area is excluded by the PACS $160 \mu \mathrm{m}$ detection limit for the GOODS-S sample.

(A color version of this figure is available in the online journal.)

dashed line and its intermediate slope of $\sim-0.5$ appears to be approximately parallel to the blue/red (low/high $v L_{v}(8) /$ LIR) separation line. In this interpretation, galaxies with constant $v L_{v}(8) /$ LIR thus appear along this mass-dependent $\operatorname{SSFR}\left(M_{*}\right)$ of the main sequence, rather than lines of constant SSFR or constant LIR. While lines of $v L_{v}(8) /$ LIR seem to be closest to the slope of the main sequence, in practice over this limited mass range and the scatter in $v L_{v}(8) /$ LIR, binning the galaxies with respect to the main sequence (slope -0.5 ), constant SSFR lines ( 0 slope), or constant LIR lines ( -1 slope) will not produce drastically different selections in terms of $v L_{v}(8) / \mathrm{LIR}$.

We quantify the latter statement via a Spearman rank correlation test of $v L_{v}(8) /$ LIR against LIR, SSFR, and the offset from the main sequence at the given galaxy mass $\Delta \log (\mathrm{SSFR})_{\mathrm{MS}}=$ $\log (\mathrm{SSFR})-\log (\mathrm{SSFR})_{\mathrm{MS}}$. The results are plotted in Figure 3 . All three tests reject the null hypothesis of uncorrelated data with high significance, but the best correlation coefficient and highest significance of the three is with the distance from the main sequence. We note that in case of a perfect correlation of $v L_{v}(8) / \mathrm{LIR}$ and $\Delta \log (\mathrm{SSFR})_{\mathrm{MS}}$, significant correlation would also be expected with LIR and SSFR over the range studied, given the definitions of these quantities.

We thus found a tendency of galaxies with similar SEDs to lie along sloped lines in the SSFR versus mass diagram. This tendency corresponds to the best correlation found between $v L_{v}(8) / \mathrm{LIR}$ and $\Delta \log (\mathrm{SSFR})_{\mathrm{MS}}$. It is consistent with the Rodighiero et al. (2010) main-sequence slope, within the statistics of this small sample. The slope of these lines as opposed to a constant SSFR makes also the correlation of $v L_{v}(8) / \mathrm{LIR}$ with LIR significant, even though it is not the best relation of the three. The physical origin of the correlation between SED shape and LIR hence seems more indirect, not fundamental as we shall discuss further in Section 9. Still, for practical purposes this correlation can be used to measure LIR from $24 \mu \mathrm{m}$ fluxes in cases where the mass is not well determined, or unknown.

In the following sections, we will explore the mid- to far-IR SEDs in two ways: in the distance from the main sequence that 


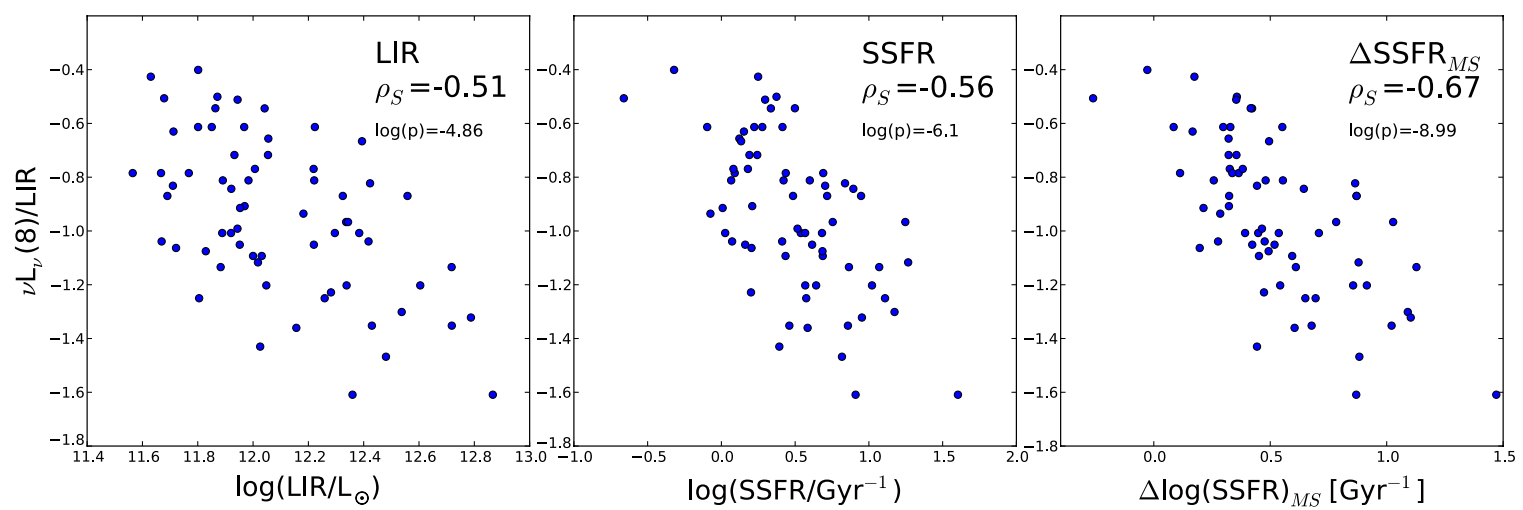

Figure 3. Spearman rank correlation test of $v L_{v}(8) /$ LIR against LIR, SSFR, and SSFR distance from the main sequence. The correlation coefficient $\rho_{s}$ and the null hypothesis probability ( $p$-value) are indicated in each panel.

(A color version of this figure is available in the online journal.)

seems to be the more fundamental relation, and the classical and simpler dependence on LIR.

\section{METHOD: MEAN SED REDSHIFT SCAN}

The study of galaxy SEDs in the mid- to far-IR range can be broken into two parts: the shape of the SED and the overall amplitude, i.e., the total LIR, defined as the total integrated luminosity between 8 and $1000 \mu \mathrm{m}$.

For studying the SED shape, one must overcome a few difficulties. The first is that due to various reasons, many of the galaxies are not detected at all wavelengths. The nondetections must be taken into account in order not to bias the results. The second is that at different redshifts the filters sample different rest-frame wavelengths. Normally this requires some inter/extrapolation to common wavelengths, which require assumptions on the same region of the SED we wish to study. This is most problematic in the mid-infrared where there are strong spectral variations on scales smaller than the filter width.

We wish to study the mean properties of mid- to far-IR emission. Therefore, instead of fitting a template SED to every individual galaxy, we take a slightly different approach: we fit a template to the combined photometric data points of a galaxy population in their rest frame. This treats the combined data as if originating from a single mean galaxy, observed by many different filters which are slightly shifted in their central wavelengths. In this method, sampling a narrow yet non-negligible redshift interval requires no interpolation/extrapolation to a common rest-frame wavelength. Such extrapolations can be problematic in the mid-infrared due to PAH features. It also does not force the SEDs to cross at a single wavelength chosen for the normalization, and the sampling of slightly different rest-frame wavelengths by the filters improves the effective resolution.

The first and most important piece of information we need for each galaxy is its LIR. Herschel-PACS, especially with the $160 \mu \mathrm{m}$ filter, allows us to accurately determine the LIR almost independently of the SED shape. Generally, in our fields and at the redshifts of interest, out of the three PACS bands, $160 \mu \mathrm{m}$ has the highest detection rate. It also samples close to $60 \mu \mathrm{m}$ rest frame at $z \sim 2$, which leads to a very robust LIR determination as Elbaz et al. (2010) demonstrated. The $60 \mu \mathrm{m}$ rest frame is a "sweet spot" for monochromatic LIR determination and we expand on this point further in Appendix A. For the $1.5<z<$ 2.5 sample, we measure LIR by fitting CE01 templates to $160 \mu \mathrm{m}$ flux alone, while preserving the library LIR-template relation (monochromatic fitting), even in cases where shorter wavelength fluxes are available. At $0.7<z<1.3$, the PACS $160 \mu \mathrm{m}$ filter has moved farther away from the rest-frame $60 \mu \mathrm{m}$ sweet spot and PACS $100 \mu \mathrm{m}$ filter has moved closer. For galaxies at these lower redshifts, we fit the best combination of CE01 template shape and scale (two free parameters) to the 100 and $160 \mu \mathrm{m}$ points simultaneously, except for a few cases at the lowest luminosities where only $160 \mu \mathrm{m}$ is available. In the latter case a monochromatic fit to LIR is used. For further discussion on the LIR determination we refer the reader to Appendix A.

After determining the LIR of each galaxy in our full sample, we are able to bin the sample by SSFR offset from the main sequence or by LIR. For each bin subsample, we fit a single template to the $v L_{v} /$ LIR values of all sources combined. Dividing all filter luminosities of a given galaxy by its own LIR serves as normalization and does not affect the flux ratios. Each galaxy thus contributes nearly equally to the overall $v L_{v} /$ LIR template fit, only depending on its photometry errors, but independent of its absolute luminosity. As a working example, we start by selecting $1.5<z<2.5$ PACS $160 \mu \mathrm{m}$ sources according to their $\Delta \log (\mathrm{SSFR})_{\mathrm{MS}}$ distance from the main sequence in the SSFR- $M_{*}$ plane. LIR is converted to SFR using the relation from Kennicutt (1998a), converted to a Chabrier IMF (Chabrier 2003):

$$
\left(\frac{\mathrm{SFR}}{M_{\odot} \mathrm{yr}^{-1}}\right)=1.09 \times 10^{-10}\left(\frac{\mathrm{LIR}}{L_{\odot}}\right) .
$$

The exact parameterization of the main sequence of SFGs is somewhat ambiguous. Different studies (at different wavelengths and using different selections of SFGs) find different slopes $n$ and a corresponding scaling $\Omega_{\mathrm{MS}}$ for the relation

$$
\log \left(\frac{\dot{M}_{*}}{M_{*}}\right)=n \log \left(\frac{M_{*}}{M_{\odot}}\right)+\log \left(\Omega_{\mathrm{MS}}\right) .
$$

In this study, we use the main sequence as determined by Rodighiero et al. (2010, corrected to a Chabrier IMF) that was derived from PEP data in GOODS-N: $\left(n, \log \left(\Omega_{\mathrm{MS}}\right)\right)=(-0.496,5.243)$ for $z \sim 2$ and $\left(n, \log \left(\Omega_{\mathrm{MS}}\right)\right)=(-0.394,3.945)$ for $z \sim 1$. The redshift of 1 is between two bins in Rodighiero et al. (2010) and we adopt the simple mean of the main sequence above and below it.

We divide our $1.5<z<2.5$ sample into six bins as illustrated in Figure 4, top panel. The bins are numbered 1-6 with increasing $\Delta \log (\text { SSFR })_{\text {MS }}$. Also indicated is the region below a line with slope -1 that is excluded by the $160 \mu \mathrm{m}$ 

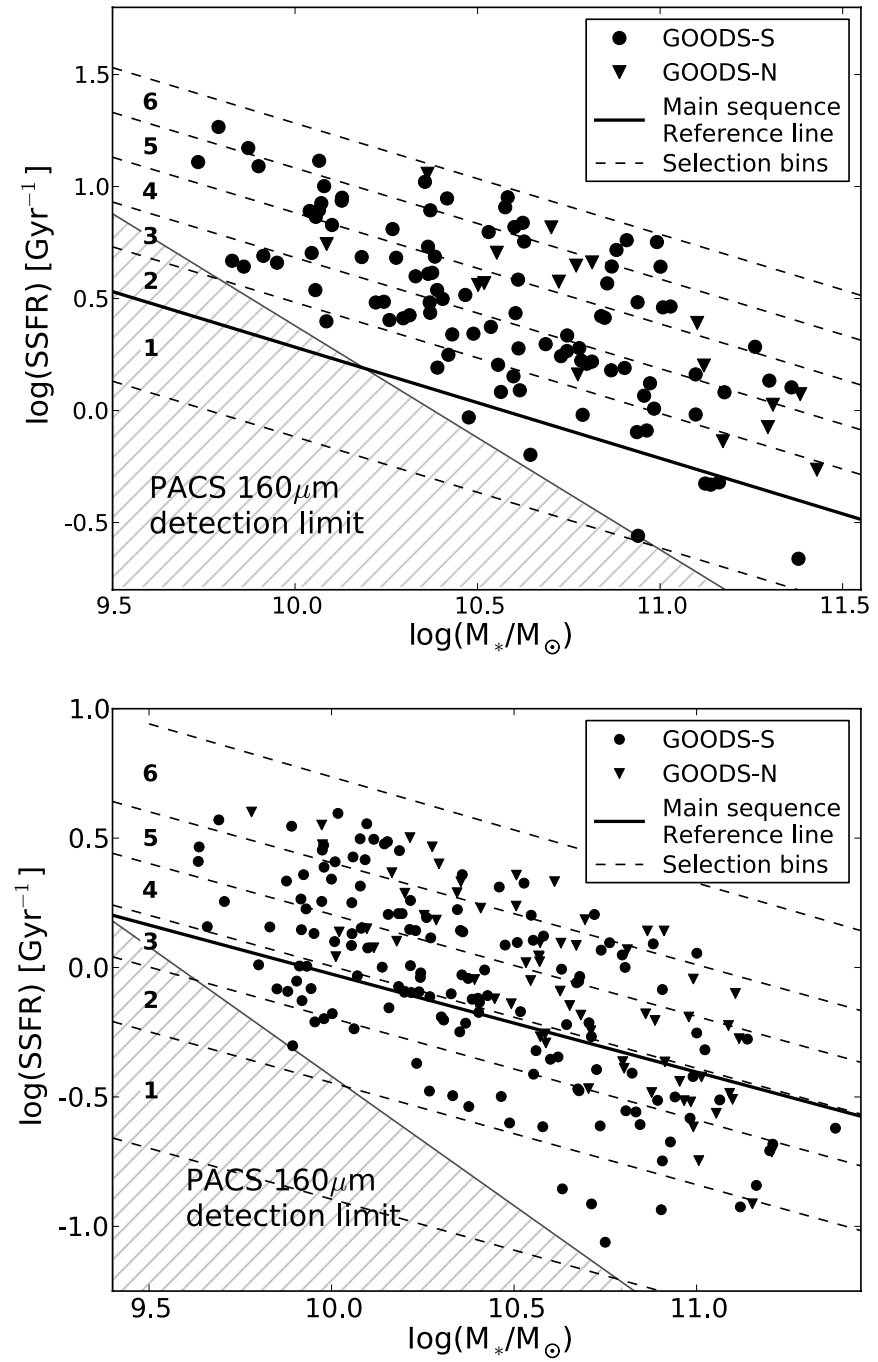

Figure 4. Top: the binning of the $1.5<z<2.5$ sample according to the distance from the main sequence. Bottom: similar to above for the $0.7<z<1.3$ sample.

flux limit. The combined photometry for each $\Delta \log (\text { SSFR })_{M S}$ bin is plotted in Figure 5, top. Each filter flux is converted to $v L_{v} /$ LIR units according to the corresponding galaxy LIR, and the data points are color coded according to their filter. Stacks of (individually) undetected 100 and $70 \mu \mathrm{m}$ (but detected at $160 \mu \mathrm{m}$ ) sources are also plotted in the respective filter color. On top of each stack point, we indicate the number of sources in the stack, and the error bars indicate the uncertainty on the mean $v L_{v} /$ LIR in the stack. For details about the stacking procedure see Appendix B.

An SED template is then fitted to the combined photometry of all galaxies in each bin using $\chi^{2}$ minimization. Since we are not making repeated measurements of the same galaxy, but rather of a galaxy population, the intrinsic scatter in the population must be taken into account when calculating $\chi^{2}$. This has a significant effect on the relative weight of each photometric point and the relative weight of the stacks. More details about the $\chi^{2}$ minimization are available in Appendix C. We would like to keep the description of the SED shapes as simple as possible. Due to its popularity, its use in determining LIR and the simple way in which it is defined, we choose to use the CE01 library. Magnelli et al. (2009) also found that at $z \sim 1$ this library reproduces the $24 / 70 \mu \mathrm{m}$ colors better than a few other popular libraries. Even though we concentrate on CE01, we will supply the means to calibrate any other library. The templates in the CE01 library represent the mid-infrared to submillimeter SEDs of local galaxies according to their total IR luminosity and are uniquely identified by their LIR. We also use them below as SED shape templates that are encoding relative ratios between the far- and the mid-infrared, and the PAH features in particular, without enforcing the locally calibrated link to total IR luminosity. To avoid confusion, we refer in this case to the template identifier as $\Lambda_{\mathrm{CE} 01}$ in units of $L_{\odot}$, irrespective of the IR luminosity to which it might have been rescaled.

We thus search the CE01 library for the best-fitting template shape. Since both data and templates are in $v L_{v} /$ LIR units, there is no scaling involved in this fit. We split at $15 \mu \mathrm{m}$ rest frame and fit the mid- and far-infrared independently. This split differentiates two distinct wavelength and physical regions: the mid-infrared is dominated by $\mathrm{PAH}$ emission from cold dust regions, silicate absorption, and possible continuum from AGN-heated hot dust, while the far-infrared peak is dominated by graybody emission of dust. In the middle, in the range of 15-60 $\mu \mathrm{m}$ (probed by PACS), a tail distribution of hotter dust temperatures and transient heating of small grains shape the SED slope (e.g., Desert et al. 1990).

In each panel of Figure 5, we indicate the best-fitting midand far-infrared $\Lambda_{\mathrm{CE} 01}$ templates. The best-fit SED is plotted as a solid black line. Broadband filter fluxes represent the weighted emission from a significant wavelength interval. For each filter, we indicate with a solid colored line the expected filter flux for the fitted template by gradually redshifting the fitted template in the range $1.5<z<2.5$ and applying the filter transmission to the template. The central wavelength of the filter scans a corresponding range of rest-frame wavelengths (redshift scan). The distinction between the template and the curve of expected filter flux is most important in the mid-infrared region.

One final note should be made: since we require PACS $160 \mu \mathrm{m}$ detections in order to estimate LIR, sources undetected in this filter are excluded. We have argued above and in Appendix A that $160 \mu \mathrm{m}$ is a good proxy for LIR at these redshifts over a wide range of SEDs. When later binning by LIR (Section 7), non-detections at $160 \mu \mathrm{m}$ thus do not introduce significant biases as we operate in narrow LIR bins: the near independence of rest-frame $v L_{\nu}(60 \mu \mathrm{m}) / \mathrm{LIR}$ from SED shape means that non-detections are mostly due to limiting luminosity distance for a given LIR. When binning by $\Delta \log (\mathrm{SSFR})_{\mathrm{MS}}$, this translates into a more complex combination of limiting luminosity distance and $M_{*}$. Unless there is an added dependence of SED shape on the absolute mass, which is not a result of the SFR $-M_{*}$ relation, this binning does not introduce biases as well.

\section{THE MID- TO FAR-IR SED AS A FUNCTION OF DISTANCE FROM THE MAIN SEQUENCE}

In Section 3.1, we found that galaxies with similar $v L_{v}(8) /$ LIR tend to lie parallel to the main sequence in their SSFR, and Section 4 described our method to buildup mean SEDs for $z \sim 2$ galaxies as a function of offset from the main sequence. We follow a similar procedure for our $0.7<z<1.3$ sample. At $z \sim 1$, the MIPS24 filter samples rest-frame wavelengths $\lambda \sim 12 \mu \mathrm{m}$, which cover entirely different spectral features than those at $8 \mu \mathrm{m}$. We therefore add the $16 \mu \mathrm{m}$ IRS blue peak-up filter data from Teplitz et al. (2011) that sample $8 \mu \mathrm{m}$ rest frame. The sample is divided into six $\Delta \log (\mathrm{SSFR})_{\mathrm{MS}}$ bins as illustrated in Figure 4, bottom panel, and SEDs are again fitted 


\section{$1.5<z<2.5$}
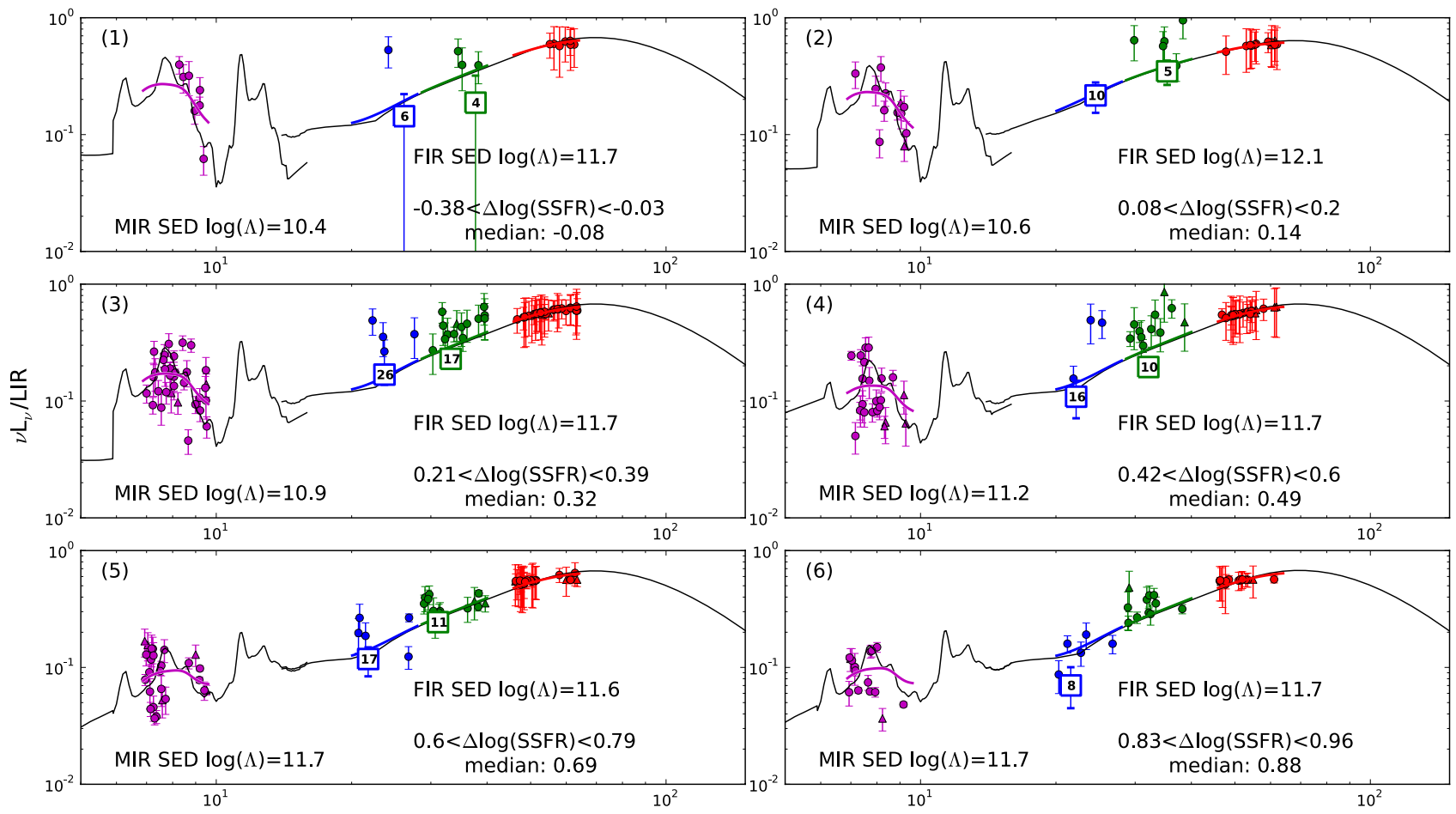

Rest Wavelength $[\mu \mathrm{m}]$

\section{$0.7<z<1.3$}
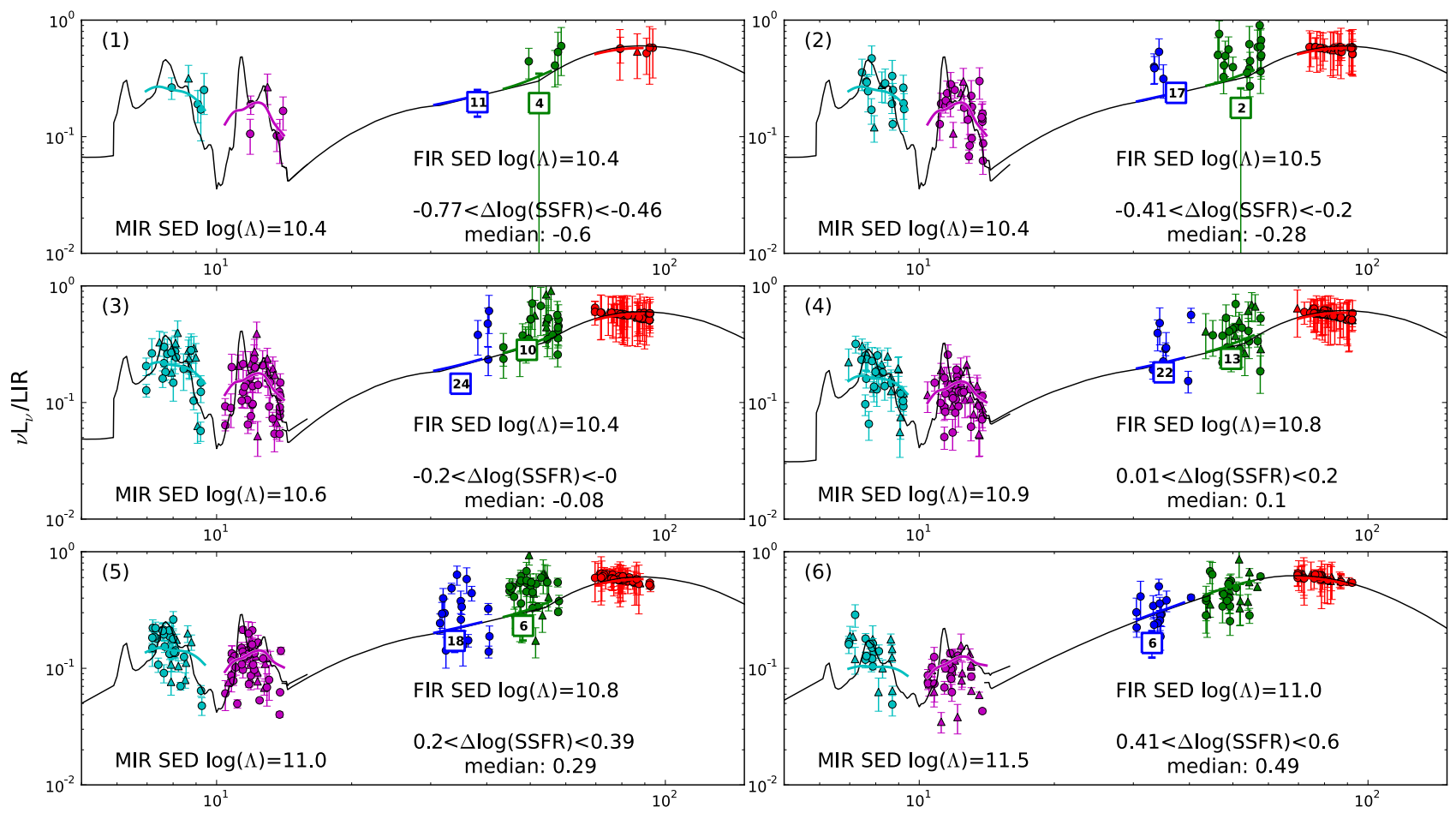

Rest Wavelength $[\mu \mathrm{m}]$

Figure 5. Top: mean SEDs fitted to the $1.5<z<2.5$ sample, binned by the $\Delta \log (\mathrm{SSFR})_{\text {MS }}$ distance from the main-sequence line. The number on the corner of each panel indicates the corresponding $\Delta \log (\mathrm{SSFR})_{\mathrm{MS}}$ bin in Figure 4, top panel. Circles and triangles are for GOODS-S and GOODS-N sources, respectively. Squares mark stacks of GOODS-S undetected sources with the number of stacked sources indicated on them. The colors red, green, blue, and purple correspond to the filter wavelengths PACS 160, 100, 70, and MIPS $24 \mu \mathrm{m}$, respectively. CE01 library SED template shapes $v L_{v} /$ LIR are fitted to the sample separately for the mid- and far-infrared rest wavelength ranges below and above $15 \mu \mathrm{m}$. These fits are plotted in solid black and the best-fitting SED shapes are labeled by their nominal luminosity $\Lambda$. The $\log (\Lambda)$ template identifier of the best-fitting templates are noted separately for the mid- and far-infrared. The expected filter fluxes for the best-fit template are plotted as thick lines matching the filter color. Bottom: same as above for the $0.7<z<1.3$ sample. The additional $16 \mu$ m photometry is colored in cyan. The panel numbers match the bins plotted in Figure 4, bottom panel.

(A color version of this figure is available in the online journal.) 


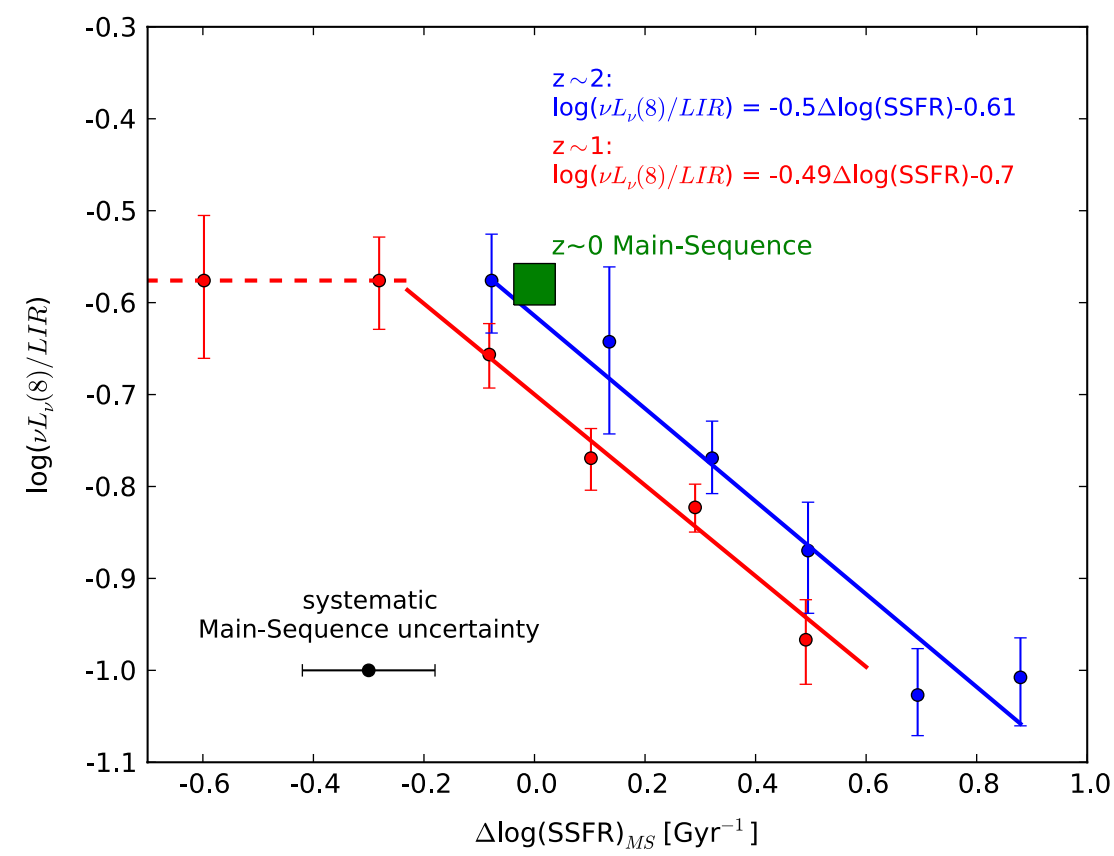

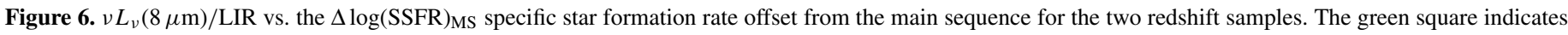

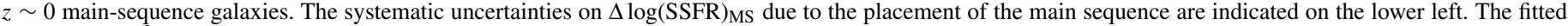
relations use $\chi^{2}$ minimization. $v L_{v}(8)$ value is the MIPS24 filter luminosity as would be measured for a galaxy at $z=2$.

(A color version of this figure is available in the online journal.)

to the combined photometry of all galaxies in each bin. The fit results are plotted in Figure 5, bottom.

For both $z \sim 2$ and $z \sim 1$ (Figure 5, top and bottom, respectively), in the sequence of increasing bin number and SSFR, there is a clear trend toward fitting the mid-infrared ratio by template shapes corresponding to higher luminosities $\Lambda_{\text {CE01 }}$, implying lower $\log \left(v L_{v}(8) /\right.$ LIR). In Section (5.1), we will quantify these effects.

In contrast, the far-infrared $\lambda>15 \mu \mathrm{m}$ is fitted by similar templates in all six bins of the $z \sim 2$ sample. In the CE01 library all templates in the range $11<\log \left(\Lambda / L_{\odot}\right)<12.1$ are nearly identical in the rest-frame wavelengths covered by the three PACS bands (20-60 $\mu \mathrm{m}$, see also Figure 14 in Appendix A) when plotted in $v L_{v}(\lambda) /$ LIR scale. This part of the far-infrared SED, among this range of templates, is fairly insensitive to the exact location of the SED peak. For the $z \sim 2$ sample, we hence cannot reliably infer dust temperatures and which template will be the most accurate in extrapolating to longer rest-frame wavelengths. We note, however, that the templates fitted to the mid-infrared $(\lambda<15 \mu \mathrm{m})$ also fit the longer wavelengths up to $60 \mu \mathrm{m}$ at $z \sim 2$. For the $z \sim 1$ sample, the bestfit templates for the far-infrared up to $\sim 90 \mu \mathrm{m}$ rest frame are close to the templates fitted to the mid-infrared, as indicated by their respective $\Lambda_{\mathrm{CE01}}$. Within the degeneracy of the templates we thus find consistent fit results for the mid- and far-infrared, but do not strongly constrain the shape of the rest far-infrared peak given the rest wavelength coverage of the PACS data.

\subsection{Building an SSFR-scaled SED Library}

We wish to derive a recipe for calibrating the CE01 SEDs by offset from the main sequence. Figure 6 concentrates all the essential mid-infrared information from the template fittings described above into one figure and presents our main result. It plots the $\nu L_{v}(8 \mu \mathrm{m}) / \mathrm{LIR}$ versus the $\Delta \log (\mathrm{SSFR})_{\mathrm{MS}}$ deviation from the main sequence. For each template fitted to the mid- infrared photometry in Figure 5, we derive the mean $v L_{v}(8) / \mathrm{LIR}$ as observed through the MIPS $24 \mu \mathrm{m}$ filter at $z=2$ (centered on $8 \mu \mathrm{m}$ rest frame). This scaling curve can be used to recalibrate any SED library (see Appendix D). Filter convolved flux is used instead of the monochromatic template point at $8 \mu \mathrm{m}$ because our template calibration is photometric in a structured region of the SED. We cannot be sure that the template is correct in its higher resolution details, which may also vary in other libraries, but note good agreement of PAH dominated CE01 template shapes with IRS spectra for a subset of our sample (Section 6). For $z \sim 1$, even though the $8 \mu \mathrm{m}$ was observed by the $16 \mu \mathrm{m}$ filter, we again specify $v L_{v}(8) / \mathrm{LIR}$ in terms of the $z=2.0 \mathrm{MIPS}$ $24 \mu \mathrm{m}$ flux for the template.

The resulting curves of $v L_{v}(8) / \mathrm{LIR}$ as a function of $\Delta \log (\mathrm{SSFR})_{\mathrm{MS}}$ for the two redshifts bins are plotted in Figure 6. The slopes of the $z \sim 2$ and $z \sim 1$ samples for $\Delta \log (\mathrm{SSFR})_{\mathrm{MS}} \gtrsim 0$ are practically identical and the curves agree within the data uncertainty (plotted error bars), and in particular the added systematic ambiguity of placement of the main sequence itself at different redshifts. For our adopted main sequence from Rodighiero et al. (2010), we estimate this placement to carry an uncertainty of $\sim 0.1-0.15$ dex, also considering slightly different methods to derive LIR. This main-sequence ambiguity results in horizontal shifts of the curves and can account for the $\Delta \log (\mathrm{SSFR})_{\mathrm{MS}}$ shift between the $z \sim 1$ and $z \sim 2$ curves.

Galaxies that are on the main sequence at the two redshift bins have nearly the same $\nu L_{v}(8 \mu \mathrm{m}) / \mathrm{LIR}$ ratio, and this ratio changes with the distance from the main sequence in a similar fashion. More importantly, it means that the increase with redshift of the SSFR at a given mass (i.e., the scaling of the main sequence with redshift) is the same as the scaling that affects SED shapes.

The clear negative slope indicates a suppression of $8 \mu \mathrm{m}$ emission versus the LIR for galaxies with SSFR higher than the main sequence. Quantitative details of this result are sensitive 
to the slope and in particular the scaling of the adopted main sequence which vary between different studies. Adopting a different main sequence will change the absolute calibration derived here, but not the fundamental behavior, namely, the saturation of $\log \left(v L_{v}(8) / \mathrm{LIR}\right)=-0.58 \mathrm{on} /$ below the main sequence. It will hardly change the slope of the curves in Figure 6.

For objects with SSFR below the main sequence, which are available only in the $z \sim 1$ sample, $\log \left(v L_{v}(8) / \mathrm{LIR}\right)$ saturates into a constant value of $-0.58 \pm 0.04$. This is the typical (near constant) value for templates in the CE01 library for local galaxies of $\log \left(\mathrm{LIR} / L_{\odot}\right)<10.2$. In the CE01 library, $\log \left(v L_{v}(8) / \mathrm{LIR}\right)$ was constrained by comparing ISOCAM-LW2 $6.7 \mu \mathrm{m}$ (also covering the $7.7 \mu \mathrm{m}$ PAH band) with the total LIR (Figure 3 in Chary \& Elbaz 2001). The correlation is almost linear up to $\log \left(\mathrm{LIR} / L_{\odot}\right) \approx 10$ and then breaks toward progressively reduced $v L_{v}(6.7) / \mathrm{LIR}$ with increasing LIR. A characterization of $\log \left(v L_{v}(8) / \mathrm{LIR}\right)$ as a function of main-sequence offset $\Delta \log (\mathrm{SSFR})_{\mathrm{MS}}$ for local galaxies is beyond the scope of this work, but we can safely assume that the normal galaxies below the $\log \left(\mathrm{LIR} / L_{\odot}\right) \approx 10$ break point for $v L_{v}(8) / \mathrm{LIR}$ in CE01 are main-sequence star formers. We use their $\log \left(v L_{v}(8) / \mathrm{LIR}\right)$ to indicate the location of the $z=0$ main sequence in Figure 6 (green square). The breakpoint luminosity translates into an SFR of $\sim 1 M_{\odot} \mathrm{yr}^{-1}$, which at $z=0$ means $\sim 10^{10} M_{\odot}$ main-sequence galaxies (Brinchmann et al. 2004; Peng et al. 2010). Due to the specifics of the CE01 sample, higher luminosities are mostly sampled by very luminous $z=0$ ULIRG mergers for which $8 \mu \mathrm{m}$ emission is suppressed. With masses of a few $10^{10} M_{\odot}$ (e.g., Dasyra et al. 2006), these ULIRGs will be clearly above the local main sequence. This implies a downward trend in Figure 6 at positive SSFR offsets above the main sequence, also for $z=0$. Elbaz et al. (2011, their Equation (5)) find a consistent value of $\mathrm{LIR} / \nu L_{v}(8)=4$ in local main-sequence galaxies, and that this ratio holds for main-sequence galaxies up to $z \sim 2.5$.

For the $z \sim 2$ sample in Figure 6, we could not resolve the break point between sloped $\log \left(v L_{v}(8) / \mathrm{LIR}\right)$ and the likely constant value at lower $\Delta \log (\mathrm{SSFR})_{\mathrm{MS}}$. This is due to our inability to include galaxies further below the main sequence at this redshift. The lowest $\Delta \log (\mathrm{SSFR})_{\mathrm{MS}}$ point which is on and slightly below the main sequence is consistent with the $\log \left(v L_{v}(8) / \mathrm{LIR}\right)=-0.58$ limit derived from $z \sim 1$ and may already include galaxies past the break.

Figure 6 implies that there is a single relation between SED shape (i.e., $v L_{v}(8) / \mathrm{LIR}$ and the matching 8-60 $\mu \mathrm{m}$ template in general) and the SSFR offset from the main sequence that applies all the way from $z \sim 0$ to $z \sim 2$. This relation can be expressed in terms of $v L_{v}(8) / \mathrm{LIR}$ :

$$
\begin{array}{ll}
\log \left(\nu L_{v}(8) / \mathrm{LIR}\right)= & \\
-0.58_{ \pm 0.04} & : \Delta_{\mathrm{MS}}<-0.14 \\
-0.65_{ \pm 0.03}-0.50_{ \pm 0.06} \times \Delta \log (\mathrm{SSFR})_{\mathrm{MS}} & : \Delta_{\mathrm{MS}} \geqslant-0.14
\end{array}
$$

where $\Delta_{\mathrm{MS}} \equiv \Delta \log (\mathrm{SSFR})_{\mathrm{MS}}$ is expressed in $\mathrm{Gyr}^{-1}$ units and we adopt the mean from $z \sim 1$ and $z \sim 2$ for the sloped part. The errors on the fitted slope and intercept coefficients are correlated with $\rho=-0.75$. For the specific case of the CE01 library, it can be approximated by luminosity labels $\Lambda_{\mathrm{CE} 01}$ of this library:

$$
\begin{array}{ll}
\log \left(\Lambda_{\mathrm{CE} 01}\right)= & : \Delta_{\mathrm{MS}}<-0.14 \\
10.38 & : \Delta_{\mathrm{MS}} \geqslant-0.14 \\
10.58+1.44 \times \Delta \log (\mathrm{SSFR})_{\mathrm{MS}} &
\end{array}
$$

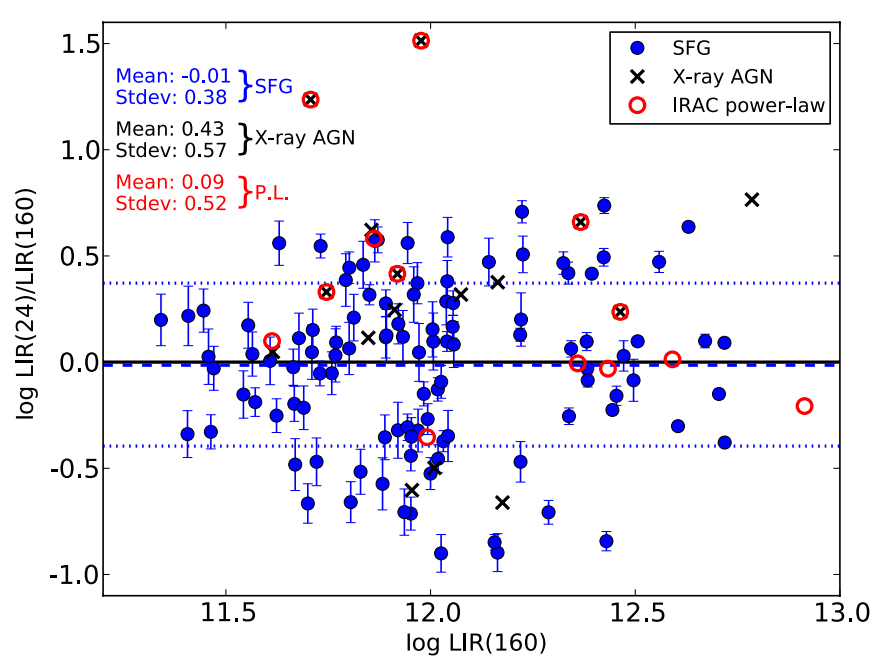

Figure 7. Scatter of LIR extrapolated for $1.5<z<2.5$ sources from observed $24 \mu \mathrm{m}$ using templates rescaled according to the source offset from the main sequence vs. LIR measured from $160 \mu \mathrm{m}$. Blue circles are SFGs, red circles are sources with power-law SED in the IRAC bands, and black crosses are X-ray AGNs. The blue dashed and dotted lines indicate the overall mean and standard deviation for the SFG.

(A color version of this figure is available in the online journal.)

We find a consistent decrease of the mid- to far-infrared ratio $v L_{v}(8) /$ LIR in the SED for galaxies that are offset above the main sequence. The strong scaling of SFR on the main sequence with redshift SFR $/ M_{*} \propto(1+z)^{2.7}$ (Bouché et al. 2010) makes LIRG- and ULIRG-like luminosities common for massive SFGs on the main sequence at $z \sim 2$. Most of these highly SFGs indeed have IR SED shapes that resemble lower redshift main-sequence galaxies. For both $z \sim 1$ and $z \sim 2$, a decrease in $v L_{v}(8) / \mathrm{LIR}$ is observed with increasing SSFR above the main sequence, but at much higher absolute luminosity than locally.

For the practical application of deriving LIR for a galaxy of known redshift, stellar mass, and rest-frame $8 \mu \mathrm{m}$ flux, we can use Equation (4) (or Equation (3) in combination with template libraries other than CE01), stellar mass, and the main sequence at the given redshift to derive a CE01 style template library as a function of LIR, which is then specific to this redshift and stellar mass (see Appendix D). This library can then be applied in the usual way to estimate LIR from the $24 \mu \mathrm{m}$ flux and redshift. We have tested this method, using it to estimate $\operatorname{LIR}(24)$ for the $1.5<z<2.5$ sources from the MIPS $24 \mu \mathrm{m}$ fluxes as described. The comparison with the LIR derived from the $160 \mu \mathrm{m}$ photometry at the far-infrared peak is plotted in Figure 7. We can see from this figure that there is no bias in derived LIR(24) and no trends with LIR, albeit the $\lesssim 0.4$ dex scatter is significant. We discuss this calibration for the use of $8 \mu \mathrm{m}$ as an SFR indicator versus other calibrations in Section 9.3.

\section{IRS SPECTRA OF HIGH- $z$ GALAXIES}

In the previous section, we have derived a calibration for the SED templates as a function of the $\triangle \mathrm{SSFR}_{\mathrm{MS}}$ distance from the main sequence. The method in which we shift the filters across the spectral features by observing galaxies of slightly different redshifts (Figure 5) can only provide limited information regarding the detailed spectrum shapes, which are consistent with the presence of PAHs. In this section, we extend this analysis using a sample of 24 IRS spectra of $z \sim 2 \log \left(\mathrm{LIR} / L_{\odot}\right) \approx 12$ and 12 spectra of $z \sim 1 \log \left(\mathrm{LIR} / L_{\odot}\right) \approx$ 

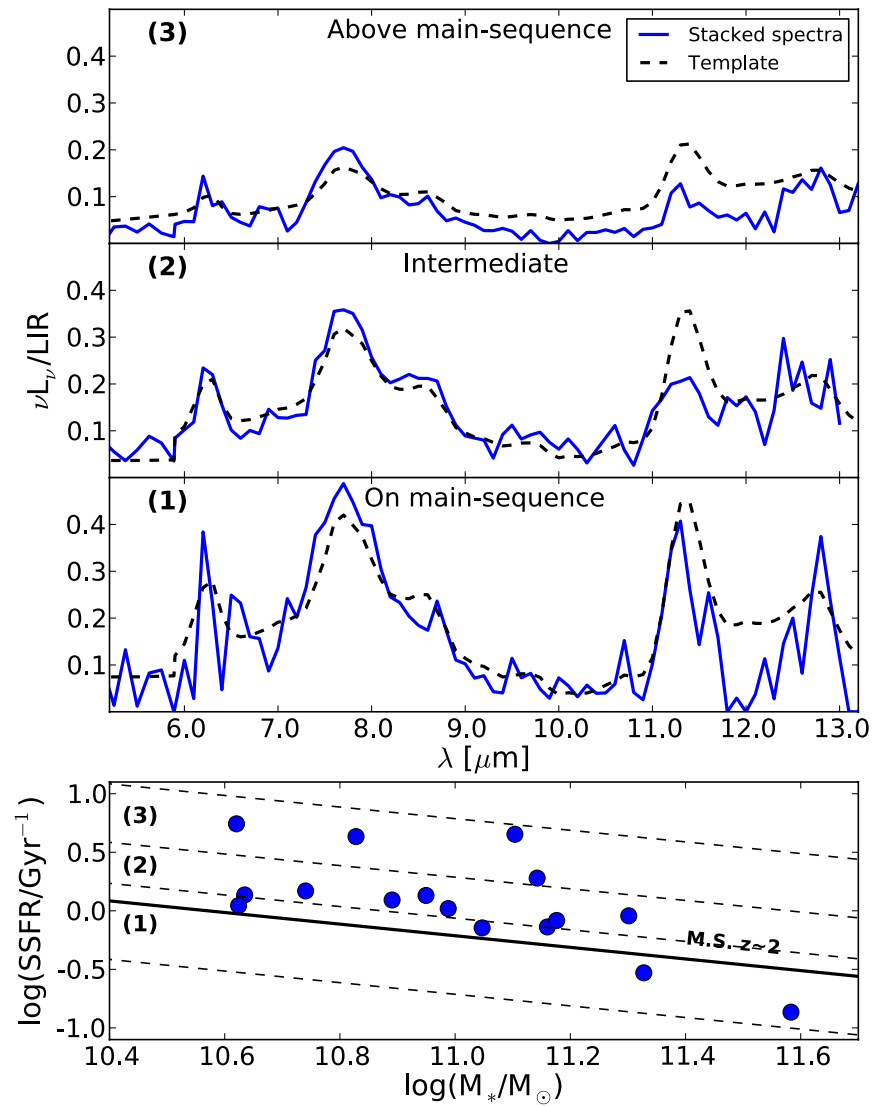

Figure 8. IRS spectra of $z \sim 2$ galaxies and associated templates. Top: template SEDs (black) scaled according to the SSFR distance from the main sequence, increasing from top to bottom, compared with stacked IRS spectra for non-AGN galaxies (blue). The three panels correspond to the selection bins as indicated by the number at the top left of each panel. The templates are scaled according to the $160 \mu \mathrm{m}$ flux and are not a fit to the spectra. Bottom: the selection bins for the stacked spectra. The bins are at a constant SSFR distance from the main sequence, which is marked as a thick solid line.

(A color version of this figure is available in the online journal.)

11 sources in our field, which was first presented by Fadda et al. (2010). In total, 31 of the IRS sources are detected by PACS at $160 \mu \mathrm{m}$ and 6 of them have an associated X-ray detection and are flagged as AGNs. The IRS spectra allow us to test the SED templates, which were assumed to have PAH features similar to those observed in local galaxies, but were calibrated above using photometry alone.

We first focus on the 16 non-AGN $z \sim 2$ objects. Due to the weak signal in each individual spectrum we stack several spectra in bins according to the distance of the galaxies from the main sequence. The bottom panel in Figure 8 shows the location of the galaxies on the SSFR versus stellar mass diagram and the three selection bins at constant distance from the main sequence. Before stacking, the spectrum of each object is converted to the $v L_{v} /$ LIR scale according to the individual LIR measured in the far-infrared by PACS. This avoids the few most luminous sources dominating the stacked result. The spectra are then averaged in $v L_{v} /$ LIR scale with equal weights. Equal weights prevent the few brightest and best signal-to-noise $(\mathrm{S} / \mathrm{N})$ sources from dominating the final mean. We then compare the stacked spectra to the modified templates as a function of SSFR mainsequence offset, as derived in Section 5. The templates are not fitted to the IRS spectrum-they are scaled by PACS $160 \mu \mathrm{m}$ flux and extrapolate down to the mid-infrared around rest $6 \mu \mathrm{m}$. The result is plotted in Figure 8.
The IRS spectra are clearly PAH dominated. This strongly argues that PAH emission rather than an AGN continuum must drive the "mid-infrared excess" and associated mid-infraredbased SFR overestimates, as noted by Fadda et al. (2010) for this sample and Rigby et al. (2008) and Murphy et al. (2009) for other IRS samples. It is also consistent with the conclusions of Elbaz et al. (2011) based on photometry. In addition, the decrease in $v L_{v}(8) /$ LIR when moving above the main sequence is indeed driven by a changing ratio of $\mathrm{PAH}$ to far-infrared, related to changing conditions in the ISM. The stacked spectra of galaxies on the main sequence or slightly below it (region 1, bottom panel) are described very well by the rescaled template for the main sequence. These galaxies with the strongest $v L_{v}(8) / \mathrm{LIR}$ relate to the ceiling value of $1 \mu \mathrm{m}$ for the $6.2 \mu \mathrm{m}$ PAH equivalent width reported in Fadda et al. (2010).

The templates were calibrated in Section 5 according to photometry which covered mainly the $7.7 \mu \mathrm{m}$ PAH peak, but in the IRS spectrum, the 6.2 and $11.3 \mu \mathrm{m}$ peaks, as well as the $10 \mu \mathrm{m}$ silicate absorption, are also well matched. The $z \sim 2$ main-sequence galaxies indeed resemble scaled-up local templates even in their spectral details: PAH emissions, silicate absorption, and continuum level. This is in broad agreement with previous results that the SEDs of high-redshift galaxies resemble scaled-up SEDs of local galaxies of lower luminosities (see the introduction). We here argue that this resemblance is based on the relation of the galaxies to the main sequence, rather than their absolute luminosities.

As we progressively move away from the main sequence (region 2 and 3 in Figure 8) the nonlinearity in the mid- to farinfrared ratio is evident. Not only does the ratio of $7.7 \mu \mathrm{m} \mathrm{PAH}$ to far-infrared decrease but the 6.2 and in particular $11.3 \mu \mathrm{m}$ emissions are fainter relative to far-infrared as well. The typical width of the main sequence at a given mass is 0.3-0.4 dex in SSFR (Noeske et al. 2007; Elbaz et al. 2007) and so bin 3 can be considered to be fully above the main sequence and shows the strongest effect. While the templates are close to the spectra around $8 \mu \mathrm{m}$ due to the way they were calibrated, they overestimate the emission from neutral PAHs at $11.3 \mu \mathrm{m}$ as well as the continuum level at wavelengths of $10 \mu \mathrm{m}$ and above. These deviations compared to the stack of four weak IRS spectra cannot be tested in the photometry because there are no filters that observe these rest-frame wavelengths at $1.5<z<2.5$.

A similar picture arises from the sample of $z \sim 1$ spectra. Nine galaxies with IR luminosity similar to local LIRGs and without an associated X-ray detection are detected by PACS $160 \mu \mathrm{m}$ and eight of them are detected at $100 \mu \mathrm{m}$ as well. We split them into two bins at $\Delta \log (\mathrm{SSFR})_{\mathrm{MS}}=0.3$ : on and above the main sequence. The stacked spectra are plotted in Figure 9. The spectra are matched very well by the scaled templates, and the relative difference in PAH strength between, on, and above the main sequence is clearly visible. The suppression of PAH strength is for this sample clearly associated with offset above the main sequence rather than higher LIR. Given the consistency of photometric and spectroscopic results, it is quantitatively expressed by Figure 6 and Equation (3). Unfortunately at this redshift, the $11.3 \mu \mathrm{m}$ PAH emission is outside the observed spectral range.

In a scenario where SFGs above the main sequence are characterized by compact intense starbursts (e.g., Wuyts et al. 2011b; Elbaz et al. 2011), two factors can contribute to the weakening of all PAHs relative to the far-infrared for these galaxies. First, high radiation field intensities in these compact regions can lead to inherently reduced $\mathrm{PAH}$ emission along with 

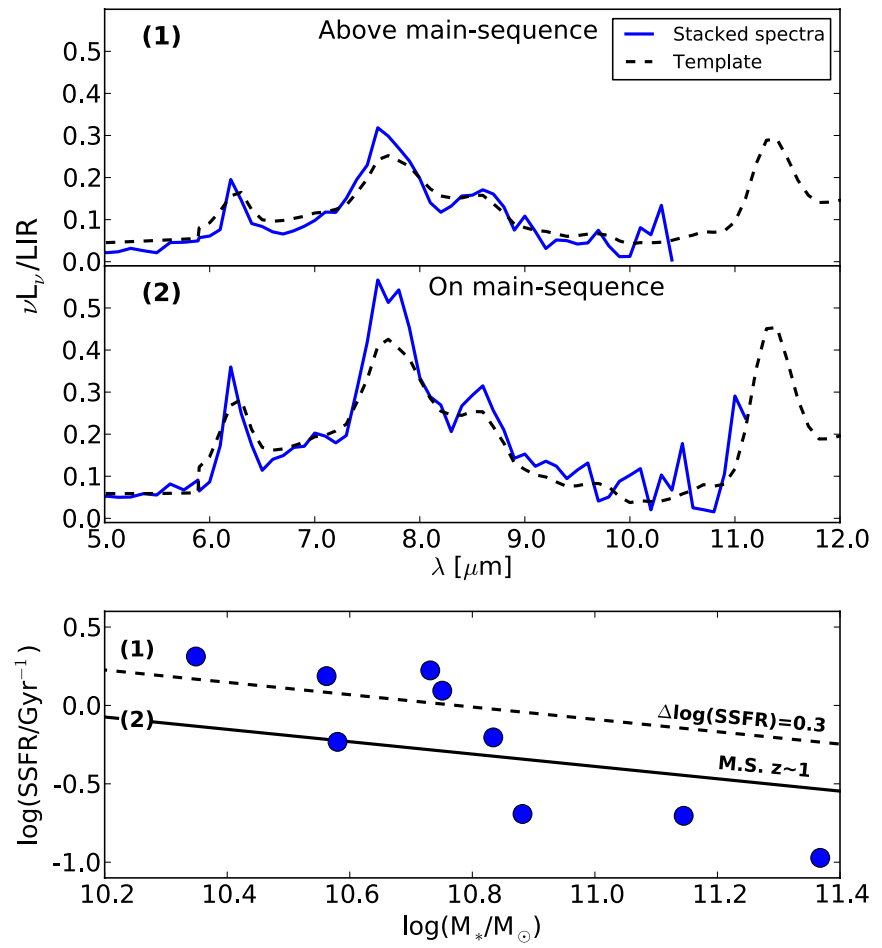

Figure 9. IRS spectra of $z \sim 1$ galaxies and associated templates. Top: template SEDs (black) scaled according to the SSFR distance from the main sequence, increasing from top to bottom, compared with stacked IRS spectra for nonAGN galaxies (blue). The two panels correspond to above and below the selection line indicated by the number at the top left of each panel and illustrated in the bottom panel. The templates are scaled according to the $160 \mu \mathrm{m}$ flux and are not a fit to the spectra. Bottom: the selection bins for the stacked spectra. The sample is split at $\Delta \log (\mathrm{SSFR})_{\mathrm{MS}}=0.3$ indicated by a dashed black line. The main sequence is marked as a thick solid line.

(A color version of this figure is available in the online journal.)

warmer large grain dust temperatures (e.g., Dale et al. 2001). Second, in the galaxies above the main sequence, the PAH emission, even in the mid-infrared, could simply be obscured by higher column densities. Given the mid-infrared extinction curve with its strong silicate feature around $\sim 10 \mu \mathrm{m}$, such obscuration of a PAH-dominated spectrum will cause reduced 8.6 and $11.3 \mu \mathrm{m}$ PAHs in the wings of the silicate feature, compared to the $7.7 \mu \mathrm{m}$ one. This is indeed observed in some local galaxies (Spoon et al. 2000). The $\mathrm{S} / \mathrm{N}$ of the stack of four objects above the $z \sim 2$ main sequence is not sufficient for such a test. From high S/N IRS spectra of local ULIRGs (thus above the main sequence), Veilleux et al. (2009) conclude that the obscuration of the PAH component is typically moderate: $A_{V} \lesssim 10$. This analogy suggests intense radiation fields to be the dominant cause of the PAH weakness, also implying warmer dust.

Kelson \& Holden (2010) argued for a strong contribution of thermally pulsating asymptotic giant branch (TP-AGB) stars to the mid-infrared emission of galaxies from $z=0$ to $z=$ 2 that could in principle be another cause for overestimated mid-infrared-based SFRs. Mid-infrared spectral evidence for AGB stars in integrated galaxy light indeed exists via detection of silicate emission in passively evolving local galaxies, with favorably low ratios of current to past star formation (Bressan et al. 2006). The IRS spectra of our SFGs instead are dominated by luminous PAHs from active star formation, arguing against a noticeable AGB contribution to their mid-infrared excess.
We conclude that the $8 \mu \mathrm{m}$ "excess" that caused the overestimation of SFR derived from MIPS $24 \mu \mathrm{m}$ photometry for $z>1.5$ galaxies was caused almost entirely by underestimation of the PAH emission when using local templates. The calibration derived in Section 5, which selects the templates by distance from the main sequence instead of LIR, correctly predicts the $8 \mu \mathrm{m}$ emission, which is dominated by the PAH emission. The spectra of the SFG (no X-ray detection or IRAC-band power law) show no significant continuum contribution which could indicate an obscured AGN, though do not exclude the presence of a low-luminosity one.

\section{THE MID- TO FAR-IR SED AS A FUNCTION OF LUMINOSITY}

In this section, we will derive a benchmark by which SED libraries can be calibrated to match high-redshift galaxies without required knowledge of the galaxy mass. We use the methods described in Section 4 but in this case using narrow LIR bins, for the same $z \sim 2$ and $z \sim 1$ parent samples.

The $1.5<z<2.5$ sample is divided into six LIR bins in the range $11.3<\log \left(\mathrm{LIR} / L_{\odot}\right)<12.8$. The $0.7<z<1.3$ sample is divided into similar LIR bins; however, we are unable to populate the two highest luminosity bins over our fields due to the rarity of such objects at $z \sim 1$. Lower luminosities of the $z \sim 1$ sample are limited by the depth of the $16 \mu \mathrm{m}$ data and in order to maintain a high $16 \mu \mathrm{m}$ detection rate, we limit the lowest luminosity similar to that of the $z \sim 2$ sample.

The result is plotted in Figure 10, top and bottom, for redshifts $z \sim 2$ and $z \sim 1$, respectively. The LIR bin range is indicated in each panel. For comparison, we also plot in dashed gray line the default CE01 template for the median luminosity in each bin. Figure 11 summarizes the mid-infrared fit results by plotting for each LIR bin the mean $v L_{v}(8) /$ LIR derived from the fitted template. For reference, we add the $v L_{v}(8) / \mathrm{LIR}$ versus LIR for the local CE01 library templates. Again we specify for all redshifts $v L_{v}(8) /$ LIR in terms of the $z=2.0$ MIPS $24 \mu \mathrm{m}$ flux for the template.

There is a clear evolution with redshift of the relation between rest-frame $v L_{v}(8 \mu \mathrm{m}) / \mathrm{LIR}$ and LIR. The local CE01 SEDs consistently underpredict the $8 \mu \mathrm{m}$ emission at all redshift and luminosity bins. This is the cause of the overestimation of SFR derived from $24 \mu \mathrm{m}$ at $z>1.5$ which was reported in previous studies, in comparison to local LIR-SED template relations. At $z \sim 1$ (Figure 10, bottom), the $24 \mu \mathrm{m}$ filter observes rest-frame wavelengths of $\lambda>10 \mu \mathrm{m}$ where the original CE01 SEDs and the newly fitted ones agree much better. This is because the region of the $11.3 \mu \mathrm{m}$ PAH with its underlying continuum scales closer to linear with LIR. Hence, the derived $24 \mu \mathrm{m}$-based SFRs were accurate for $z<1.5$ galaxies. The rest-frame $8 \mu \mathrm{m}$ emission is underpredicted by the templates at $z \sim 1$ as well, but it is not covered by the MIPS24 filter. A $16 \mu \mathrm{m}$ filter is required to detect at $z \sim 1$ an effect similar to the $z \sim 2$ "mid-infrared excess."

Interestingly, for our redshift bins the points in Figure 11 are nearly aligned and we pass a linear fit through them. The slopes of the relations for $z=2,1$, and 0 (the CE01 original library) are nearly identical. The curves for each redshift and $\log \left(L / L_{\odot}\right) \sim 12$ are

$$
\begin{array}{ll}
\log \left(v L_{v}(8) / \operatorname{LIR}\right)= & \\
-0.35_{ \pm 0.08} \log \left(\mathrm{LIR} / 10^{12} L_{\odot}\right)-0.86_{ \pm 0.03} & : z \sim 2 \\
-0.32_{ \pm 0.13} \log \left(\mathrm{LIR} / 10^{12} L_{\odot}\right)-0.96_{ \pm 0.06} & : z \sim 1 \\
-0.33 \log \left(\operatorname{LIR} / 10^{12} L_{\odot}\right)-1.13 & : z \sim 0 .
\end{array}
$$


$1.5<z<2.5$
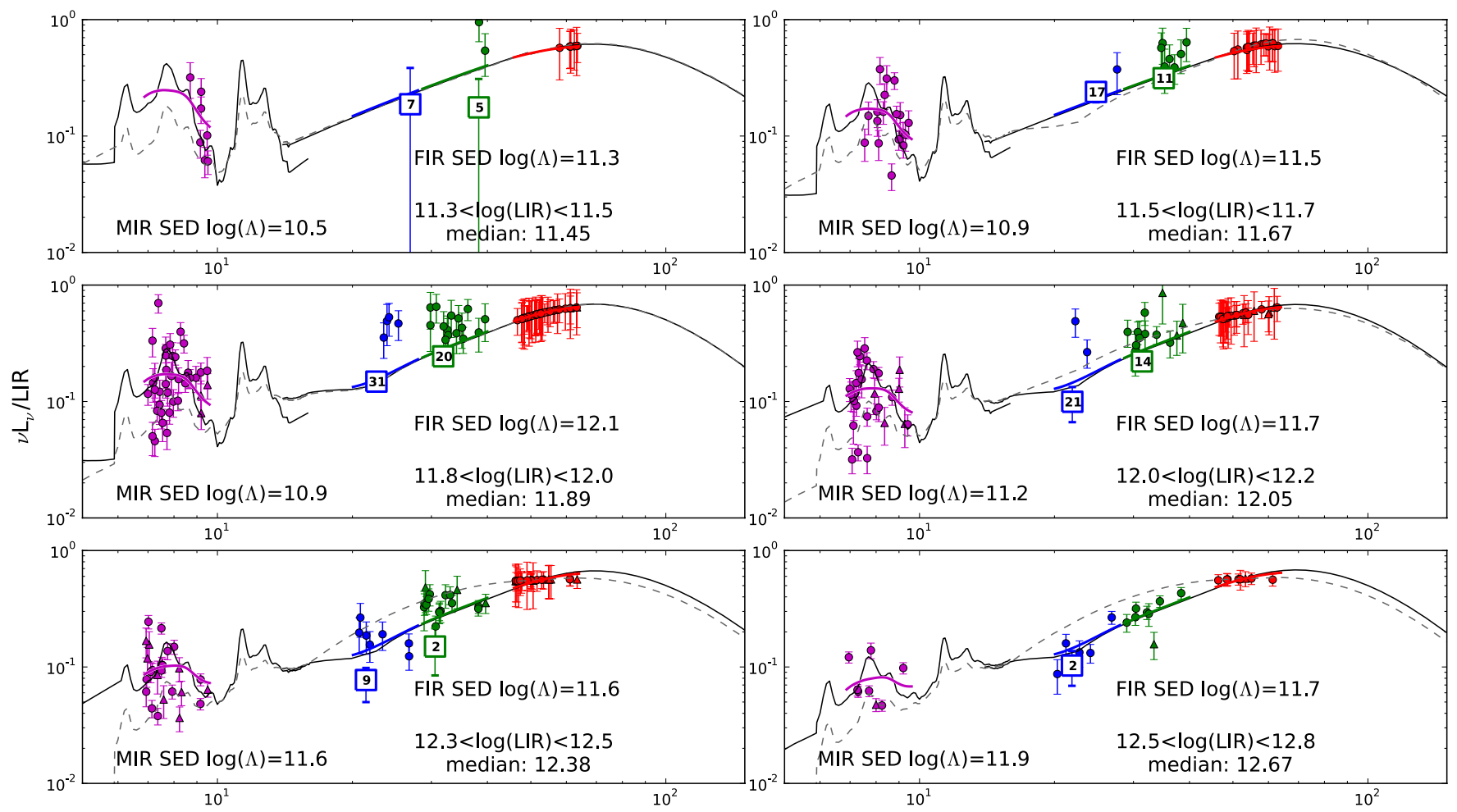

Rest Wavelength $[\mu \mathrm{m}]$

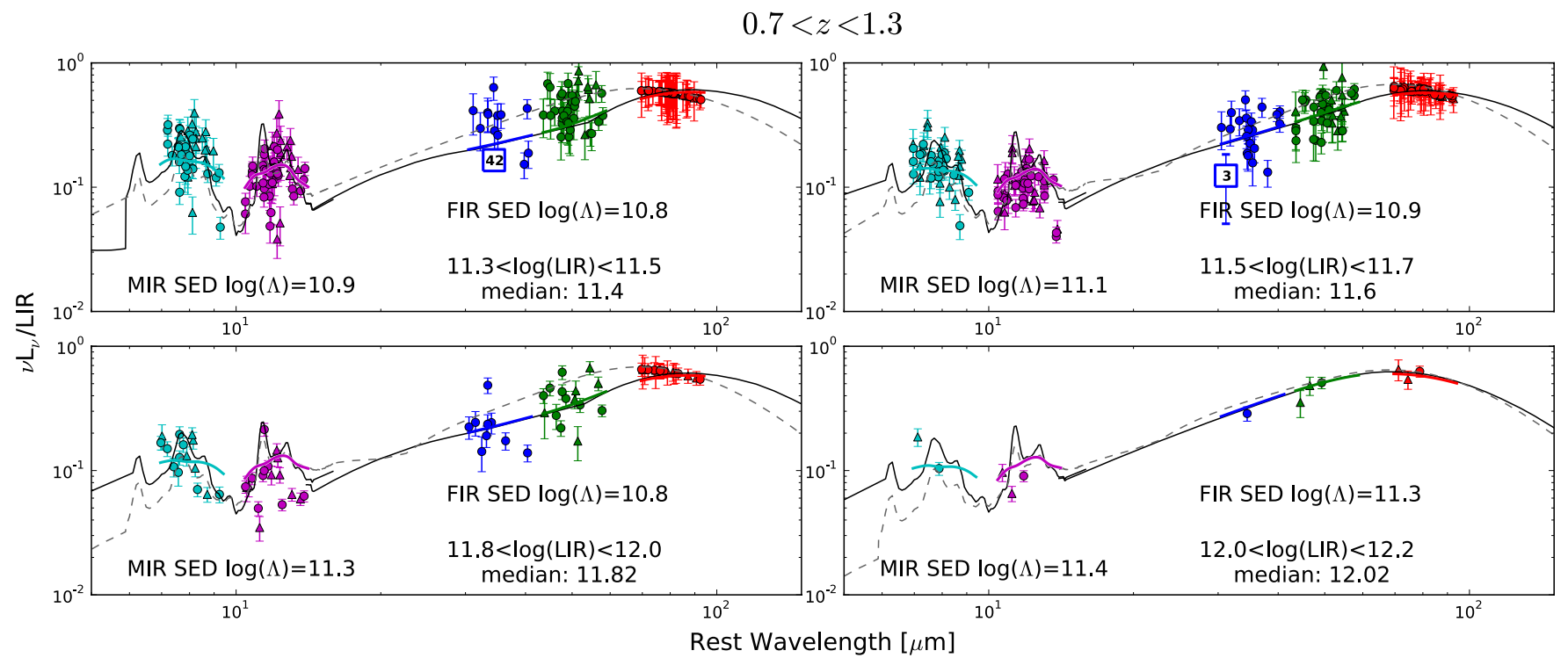

Figure 10. Top: rest-frame $v L_{v} /$ LIR for various LIR bins of $1.5<z<2.5$ galaxies. Colors and symbols are identical to those used in Figure 5 . The original CE01 template that is associated with the bin luminosity LIR is plotted as a dashed gray line. Bottom: same as above for $0.7<z<1.3$ galaxies. The additional IRS $16 \mu \mathrm{m}$ photometry is colored in cyan.

(A color version of this figure is available in the online journal.)

One can view this redshift evolution in two ways. The first is that the PAH strength $v L_{v}(8) /$ LIR increases with redshift for a given LIR, i.e., evolution upward in Figure 11. The second is that high-redshift galaxies have a $v L_{v}(8) /$ LIR similar to lower luminosity local galaxies, i.e., a shift to the right in Figure 11 by $\sim 0.5$ dex to $z \sim 1$ and $\sim 0.8$ dex to $z \sim 2$. The results of Section 5 favor the second description. This in turn predicts that the relations described in Equation (5) will flatten into the constant main-sequence value of $\log \left(v L_{v}(8) / \mathrm{LIR}\right)=-0.58$ at lower luminosities, as also happens with local galaxies. This description via a shift in the LIR associated with a given template to lower luminosities as the redshift increases is also in line with the results of previous work listed in the introduction. Thus, the overestimation of $24 \mu \mathrm{m}$ SFRs was not due to globally enhanced PAH strength at high redshifts, but due to a mismatch of template shapes with the associated LIRs in the library. Same 


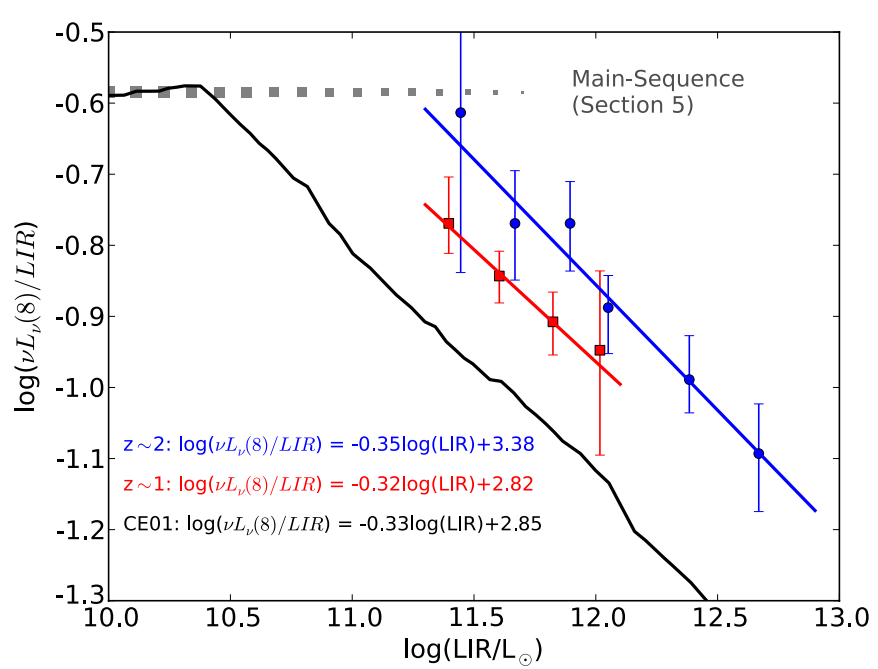

Figure 11. Mean $\log \left(v L_{v}(8) / \mathrm{LIR}\right)$ vs. $\log (\mathrm{LIR})$ for $z \sim 2$ (blue) and $z \sim 1$ (red) galaxies. The relation for local galaxies as represented by the CE01 templates is plotted in black. The gray dotted line marks the main-sequence $v L_{v}(8) / \mathrm{LIR}$ value as derived in Section 5. $v L_{v}(8)$ value is the MIPS24 filter luminosity as would be measured for a galaxy at $z=2$.

(A color version of this figure is available in the online journal.)

physical conditions in galaxies of a given LIR at all redshifts cannot be assumed. We will discuss the relation between the above description (SEDs depend on LIR) and our preferred description from Section 5 (SEDs depend on distance from the main sequence) in Section 9.

Adapting the CE01 library for use with high-redshift galaxies is a simple matter of assigning new luminosities to each template. For example, from Equation (5) we get that a CE01 template, originally associated with luminosity $\Lambda_{\mathrm{CE} 01}$, should be rescaled to a new luminosity $L_{z=2}$ with which it is associated at $z \sim 2$, using the relation

$$
\log \left(L_{z=2}\right)=0.943 \log \left(\Lambda_{\mathrm{CE} 01}\right)+1.51 .
$$

We stress that the negative slopes in Equation (5) are valid only in the luminosity range constrained by our PACS data. Locally, $\log \left(v L_{v}(8) / \mathrm{LIR}\right)$ levels off at $\sim-0.58 \mathrm{dex}$ for low-luminosity objects (Chary \& Elbaz 2001) as illustrated in Figure 11. Since low luminosities are not sufficiently constrained in our $z \geqslant 1$ data (16 $\mu \mathrm{m}$ limited) or $z \geqslant 2$ data $(160 \mu \mathrm{m}$ limited), it appears prudent to either not apply Equation (5) below the luminosities that are constrained at a given redshift or at least assume a similar leveling off at $\log \left(v L_{v}(8) / \mathrm{LIR}\right)=-0.58$, indicated by the fading dotted line in Figure 11. We compare the template calibration derived in this section with other calibrations in Section 9.3.

The far-infrared range covered by PACS, i.e., rest-frame wavelengths of $20-90 \mu \mathrm{m}$ in Figure 10, shows a behavior consistent with the scaling of the SEDs in the mid-infrared. The best-fitting templates for the $z \sim 2$ sample are in the range of $11.3<\log \left(\Lambda_{\mathrm{CE} 01}\right)<12.1$. In practice, these CE01 templates have very similar $v L_{v}(\lambda) / \mathrm{LIR}$ across the rest-frame wavelengths probed by the PACS filters and the exact $\Lambda_{\text {CE01 }}$ is degenerate in this luminosity and wavelength range. At the highest luminosities (bottom panels), local galaxies would typically have a hotter $\left(T_{\text {dust }} \sim 50 \mathrm{~K}\right)$ ULIRG-like rest-frame far-infrared SED, plotted in dashed line. The observed SEDs instead resemble LIRG-like $\left(\log \left(\mathrm{LIR} / L_{\odot}\right)>11\right)$ SED shapes with their luminosity elevated by $\sim 0.4-0.8$ dex (see also Muzzin et al. 2010; Rex et al. 2010). At the lower luminosities, the 70 and $100 \mu \mathrm{m}$ filters only detect the upper part of the population scatter. However, when taking into account the stacks (in some cases containing much larger number of sources than the detections) the mean fits the local LIRG templates. At the highest luminosities, we detect nearly the full sample at all wavelengths, and the match to the best-fit template is quite striking-almost as if we are sampling the same galaxy at different $1.5<z<2.5$ redshifts.

\section{AGNs VERSUS SFGs}

In all our samples thus far we have removed all sources detected in the Chandra 2 Ms surveys in GOODS-N and GOODS-S. The stacked IRS spectra of the SFGs (Section 6) clearly show mid-infrared emission dominated by PAHs typical of star formation and no significant emission from obscured AGNs. We now turn to inspect the mid-infrared emission of the $\mathrm{X}$-ray sources. At redshifts of 2 and with the depth of the $2 \mathrm{Ms}$ catalogs, it is safe to assume that all the X-ray sources detected by PACS $160 \mu \mathrm{m}$ are AGN hosts. Only a small fraction of the highest LIR sources are suspected to have their X-ray emission dominated by star formation (see Section 2), which does not rule out the possible presence of an AGN.

In Section 5, Figure 7, we compared the LIR as derived from $24 \mu \mathrm{m}$ to the LIR derived from $160 \mu \mathrm{m}$. In the figure, we also plotted the X-ray AGNs of our sample (black $\times$ marks) and sources that show a clear power-law SED in the 3.6-8.0 $\mu \mathrm{m}$ Spitzer-IRAC bands (red circles). If AGN-heated dust contributed significantly to the mid-infrared emission, we should expect an enhanced L24/L160 for these galaxies due to the mid-infrared continuum emission from warm circumnuclear dust. This would lead to a wrong extrapolation from mid- to the far-infrared and overprediction of the luminosity. In the figure, most AGNs are well within the scatter of the normal SFGs. A small bias does exist and AGN hosts show a small statistical enhancement in the $\sim 8 \mu \mathrm{m}$ flux. In only 2 out of 18 AGNhosting galaxies, the mid-infrared emission is significantly enhanced compared to the typical scatter for the SFG in the sample.

The Fadda et al. (2010) IRS sample includes five $z \sim 2$, X-ray AGNs which are detected with PACS $160 \mu \mathrm{m}$. These were excluded from the analysis in Section 6. The individual spectra are quite noisy. However, when normalizing by each galaxy's LIR, four of the spectra overlap within the noise, while one (U4950) shows significantly brighter mid-infrared emission. In Figure 12, we plot the stacked spectrum of the four $z \sim 2$ X-ray-detected sources from the Fadda et al. (2010) sample. We use the same stacking (spectral averaging) procedure as in Section 6. The spectrum of U4950 is plotted separately. Overplotted is the expected mean spectrum from the CE01 templates, rescaled according to the results from Section 5 and stacked in a similar way as the spectra. The templates that were scaled according to the non-AGNs also match the X-ray sources quite well. In particular, there is no enhanced continuum level that may be attributed to AGN-related circumnuclear dust emission. The stacked AGN mid-infrared spectrum shows all the typical features of an SFG and the relative AGN contribution must be low, as also concluded by Fadda et al. (2010).

The spectrum of U4950 is significantly different and hence was not included in the stack. In addition to having much higher rest mid-infrared fluxes than expected from an SFG, which as judged from the LIR lies exactly on the Rodighiero et al. (2010) main sequence (Figure 12, bottom panel), it seems to be flatter and elevated by an added continuum rather than by an enhanced 

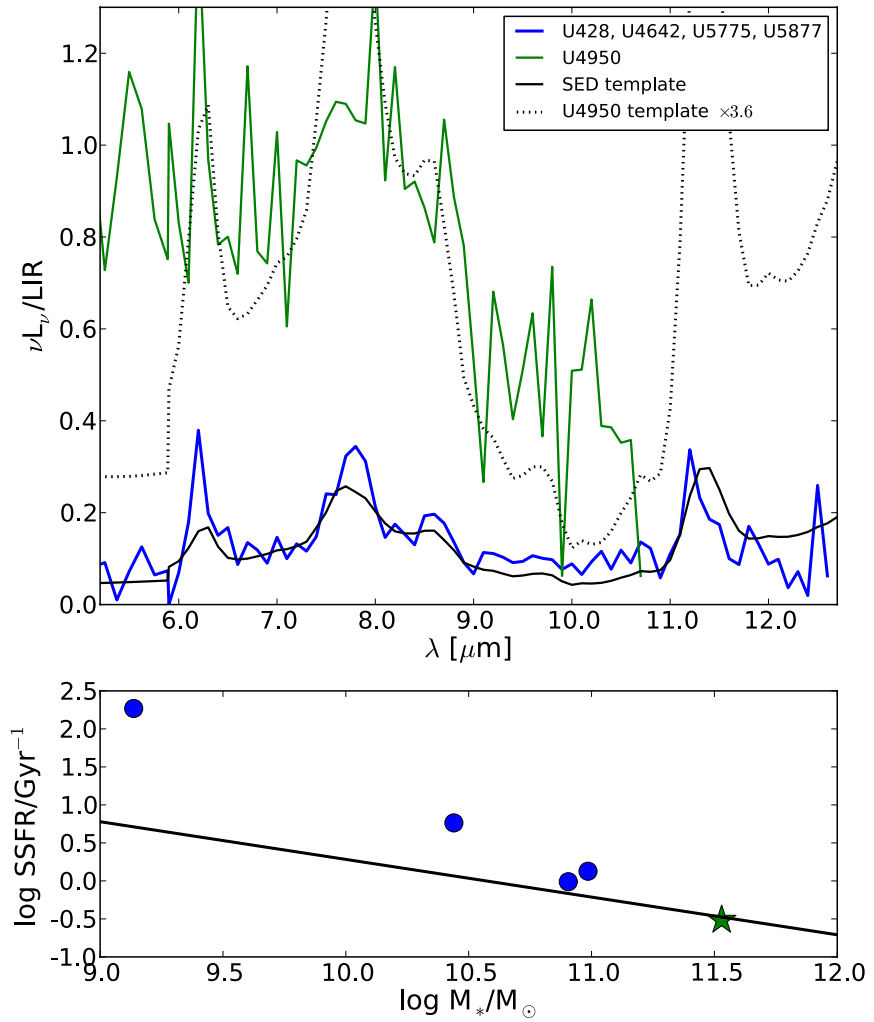

Figure 12. Top: stacked IRS spectrum of four $z \sim 2$ AGNs (blue). Spectrum of another AGN (U4950) is plotted in green. The predicted stacked spectrum from the rescaled CE01 templates is in solid black. The templates are fitted to the PACS $160 \mu \mathrm{m}$ flux, not to the spectrum. Bottom: the location of the AGN hosts with respect to the main sequence. U4950 is plotted as a green star.

(A color version of this figure is available in the online journal.)

PAH emission. The SFG template expected for U4950 according to its mass and LIR is plotted as a dotted line in Figure 12, scaled up by a factor of 3.6 to match its observed $24 \mu \mathrm{m}$ flux. The U4950 spectrum is fundamentally different from such a scaled SFG template and is dominated by AGN continuum which is evident shortward of $6 \mu \mathrm{m}$, where AGN continua can be cleanly identified (Laurent et al. 2000).

This behavior is consistent with the X-ray properties. We convert from $L_{x}(2-10 \mathrm{keV})$ to bolometric luminosity $L_{\mathrm{AGN}}$ using the relation of Maiolino et al. (2007) and a factor of seven (Netzer \& Trakhtenbrot 2007) conversion from optical to bolometric luminosity:

$$
\log \left(L_{\mathrm{AGN}}\right)=\frac{\log \left(L_{x}\right)-11.78}{0.721}+\log (7) .
$$

The IR, X-ray, and AGN bolometric luminosities are summarized in Table 4. U4950 has a significantly higher $\log \left(L_{\mathrm{AGN}} / \mathrm{LIR}\right)$ than the next highest ratio by more than $0.8 \mathrm{dex}$ and more than 2 dex higher than the rest of the AGNs in this sample.

Bauer et al. (2010) derived the following relation between mid-infrared and X-ray AGN luminosity:

$$
\log \left(\frac{L_{5.8 \mu \mathrm{m}}}{\mathrm{erg} \mathrm{s}^{-1}}\right)=1.21 \pm 0.06 \cdot \log \left(\frac{L_{2-10 \mathrm{keV}}}{\mathrm{erg} \mathrm{s}^{-1}}\right)-8.7 \pm 2.6 .
$$

This relation has been derived for much more luminous AGNs than the ones in our sample, but it is also consistent with the results of Lutz et al. (2004), which were derived for lowluminosity AGNs. Combining this relation with Equation (5)
Table 4

IRS Sources from the Fadda et al. (2010) Sample with an X-ray Detection

\begin{tabular}{lcccc}
\hline \hline ID & $\begin{array}{c}\log L_{2-10 \mathrm{keV}^{\mathrm{a}}} \\
\left(\mathrm{erg} \mathrm{s}^{-1}\right)\end{array}$ & $\begin{array}{c}\log L_{\mathrm{AGN}}{ }^{\mathrm{b}} \\
\left(\mathrm{erg} \mathrm{s}^{-1}\right)\end{array}$ & $\begin{array}{c}\log \mathrm{LIR}^{\mathrm{c}} \\
\left(L_{\odot}\right)\end{array}$ & $\begin{array}{c}\log v L_{v}(24 \mu \mathrm{m})^{\mathrm{c}} \\
\left(L_{\odot}\right)\end{array}$ \\
\hline $\mathrm{U} 428$ & $<42.43$ & $<43.36$ & 12.07 & 11.40 \\
$\mathrm{U} 4642$ & 42.54 & 43.51 & 11.86 & 11.42 \\
$\mathrm{U} 4950$ & 44.28 & 45.91 & 11.98 & 11.93 \\
$\mathrm{U} 5775$ & $<42.54$ & $<43.51$ & 12.16 & 11.28 \\
$\mathrm{U} 5877$ & 43.96 & 45.47 & 12.37 & 11.47 \\
\hline
\end{tabular}

Notes.

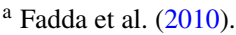

b Equation (7).

c This work.

for $z \sim 2$ and assuming that the AGN continuum is roughly flat in $\nu F_{\nu}$ between 5.8 and $8 \mu \mathrm{m}$, we get the condition that in order for a $z \sim 2$ AGN to dominate the emission close to rest-frame $8 \mu \mathrm{m}$, the relation between the X-ray luminosity and the LIR must satisfy

$$
\log \left(\frac{L_{2-10 \mathrm{keV}}}{\operatorname{erg~s}^{-1}}\right) \gtrsim \begin{cases}0.537 \log \left(\frac{\mathrm{LIR}}{\operatorname{erg~s}^{-1}}\right)+19.71 & \log \left(\frac{\text { LIR }}{L_{\odot}}\right) \geqslant 11.3 \\ 0.826 \log \left(\frac{\mathrm{LIR}}{\operatorname{erg~s}^{-1}}\right)+6.71 & \log \left(\frac{\text { LIR }}{L_{\odot}}\right)<11.3 .\end{cases}
$$

Or in terms of bolometric AGN luminosity (Equation (7))

$$
\log \left(\frac{L_{\mathrm{AGN}}}{\operatorname{erg~s}^{-1}}\right) \gtrsim \begin{cases}0.745 \log \left(\frac{\mathrm{LIR}}{\operatorname{erg~s}^{-1}}\right)+11.84 & \log \left(\frac{\mathrm{LIR}}{L_{\odot}}\right) \geqslant 11.3 \\ 1.145 \log \left(\frac{\mathrm{LIR}}{\operatorname{erg~s}^{-1}}\right)-6.18 & \log \left(\frac{\mathrm{LIR}}{L_{\odot}}\right)<11.3 .\end{cases}
$$

The condition for $\log \left(\mathrm{LIR} / L_{\odot}\right)<11.3$ assumes that $v L_{v}(8) / \mathrm{LIR}=-0.58$ below this luminosity, as suggested in Section 7. A similar, albeit more complicated relation that involves $\Delta \log (\mathrm{SSFR})_{\mathrm{MS}}$ can be derived by combining Equation (8) with Equation (3).

Overall, for our sample where $\log \left(\mathrm{LIR} / L_{\odot}\right) \sim 12$, an AGN bolometric luminosity of $\log \left(L_{\mathrm{AGN}} / \mathrm{erg} \mathrm{s}^{-1}\right)>45.8$ or $\mathrm{X}$-ray luminosity $\log \left(L_{2-10 \mathrm{kev}} / \mathrm{erg} \mathrm{s}^{-1}\right)>44.2$ is required to dominate the $8 \mu \mathrm{m}$ emission. Only U4950 reaches these AGN luminosities in the IRS sample used here and indeed shows a clear excess in its $24 \mu \mathrm{m}$ flux. In general, such high AGN luminosities are rare in the GOODS fields which explains why most of our (far-infrared bright) AGNs are indistinguishable from the SFGs in their mid- to far-IR emission (see also Nordon et al. 2010; Elbaz et al. 2010). The changed slope at $\log \left(\mathrm{LIR} / L_{\odot}\right)<$ 11.3 corresponds to a population that is dominated by galaxies on or below the main sequence. Here, the $8 \mu \mathrm{m}$ luminosity associated with the star formation decreases proportionally to LIR (constant $\left.\nu L_{v}(8) / \mathrm{LIR}\right)$, while above that threshold the $8 \mu \mathrm{m}$ luminosity will vary more slowly with LIR due to the changing mid- to far-infrared SEDs. The ratio of AGN luminosity and LIR at which the AGN dominates the $8 \mu \mathrm{m}$ emission hence clearly depends on sample selection.

Fadda et al. (2010) fitted and subtracted the PAH emission from the stacked spectra of all the IRS sources for which the mid-infrared spectra do not appear to be dominated by an AGN. They were thus able to constrain the mean AGN contribution to the $6 \mu \mathrm{m}$ continuum and derived a mean intrinsic AGN $L_{2-10 \mathrm{keV}}=1 \times 10^{42} \mathrm{erg} \mathrm{s}^{-1}$. According to the above analysis, such luminosities are nearly two orders of magnitude lower than the AGN luminosities required in order to create a true broadband $24 \mu \mathrm{m}$ "excess" in massive $\left(\sim 10^{11} M_{\odot}\right), z \sim 2$, mainsequence galaxies. Given that on average AGN hosts seem to 
have similar SFR as non-active massive galaxies at the same redshift (Shao et al. 2010), this will likely still be true for many less far-infrared luminous objects, but a higher fraction of quenched sources with $24 \mu \mathrm{m}$ emission dominated by AGNs may be expected.

\section{DISCUSSION}

\subsection{The Infrared SED Reflects ISM Conditions rather than Total LIR}

Our findings from the previous sections can be summarized as follows: the SED shape and in particular $v L_{v}(8) / \mathrm{LIR}$ correlate best with the offset from a sloped reference line in the $\operatorname{SSFR}\left(M_{*}\right)$ diagram. Over our limited mass range, the dependence on the exact main-sequence slope is weak-it is the normalization that is more important. The redshift evolution of SEDs in this picture is driven by the shift of the main sequence to higher SSFR at higher redshift. The SEDs also correlate (slightly worse and with explicit redshift dependence) with the absolute LIR, and the redshift evolution is manifested here as a shift in the LIR associated with each template shape.

If infrared SED shape is tied to a typical local radiation field intensity (e.g., Dale et al. 2001), which in turn is related to the efficiency $\mathrm{SFR} / M_{\mathrm{Gas}}(\mathrm{SFE})$ by which molecular gas is converted to stars (the number of young stars per gas mass available to irradiate it), then it will also be linked to the $\mathrm{SSFR}$, since $\mathrm{SFE}=\mathrm{SSFR} \cdot M_{*} / M_{\mathrm{Gas}}$. Because both gas fraction $M_{\mathrm{Gas}} /\left(M_{\mathrm{Gas}}+M_{*}\right)$ and main-sequence SSFR rise toward higher redshift, the increase in gas fraction and the increase in SSFR will partly compensate and SFE will be more closely linked to the SSFR offset from the main sequence. SFR $/ M_{\text {Gas }}$ is lower (by factors 4-10) in $z \sim 2$ high-redshift main-sequence galaxies, compared to local ULIRGs with similar SFR but lower total gas fractions (e.g., Genzel et al. 2010). At $z \sim 0$, radiation field intensity, SFR $/ M_{\mathrm{Gas}}$, and SSFR offset are empirically correlated with LIR, largely driven by the peculiar properties of merger driven (U)LIRGs and closely linked to their compactness, as also reflected in the local compactness/temperature relation (Chanial et al. 2007). The larger gas fractions at higher redshifts permit larger LIR before invoking special events like mergers and will shift correlations with LIR. The preferred connection between SED shape and SSFR offset from the main sequence shows that the infrared SED shape is physically linked to the local ISM conditions, and only indirectly to LIR. In line with these arguments, sizes of equivalently luminous IR galaxies change to higher redshift, with implications on their SED (Rujopakarn et al. 2011a, 2011b).

Far-infrared fine-structure emission lines and in particular [C II] exhibit a similar behavior to the PAHs. Compact luminous objects with intense radiation fields such as local ULIRGs show a [C II] deficit relative to the far-infrared continuum emission (Malhotra et al. 1997, 2001; Contursi et al. 2002; Luhman et al. 2003) with arguments for a direct physical link to PAH (Helou et al. 2001). The [C II] line with a wavelength of $158 \mu \mathrm{m}$ is much less likely to be attenuated than the mid-infrared PAHs and the deficit is intimately related to the radiation fields around the star-forming regions and the resulting structure of the $\mathrm{H}$ II and photodissociation regions (PDRs). While there are no [C II] observations for our specific targets, the qualitative findings for $[\mathrm{C} \mathrm{II}]$ both locally and at high redshift are consistent with the behavior of $v L_{v}(8) / \mathrm{LIR}$. There is a high- $z$ [C II] deficit-compared to local, it is setting in at higher LIR, but at a similar ratio of LIR and gas mass (SFE), equivalent to similar main-sequence offset (Graciá-Carpio et al. 2011). This is directly analogous to our finding of a relation $v L_{v}(8) / \mathrm{LIR}$ to LIR that evolves with redshift, but a single relation with mainsequence offset. The connection between SFE and offset from the main sequence which was described above, together with the analogy between the [C II] deficit and decreasing $v L_{v}(8) / \mathrm{LIR}$ in galaxies above the main sequence, suggest that the two observed phenomena are likely related and intense radiation fields to be the dominant cause of the PAH weakness.

To zeroth order, the redshift-dependent main sequence can be seen as a reference and with the distance from this reference a number of observables change together: the SSFR (by definition), the star formation efficiency $\mathrm{SFR} / M_{\mathrm{Gas}}$ (Genzel et al. 2010), morphology and compactness (Wuyts et al. 2011b; Elbaz et al. 2011), the mid- to far-infrared SED (this work; Elbaz et al. 2011), the far-infrared SED shape tracing the large grain temperature (Elbaz et al. 2011), and far-infrared fine-structure emission line deficits (Graciá-Carpio et al. 2011). Compactness due to mergers is likely a key factor in changing the local conditions in the ISM that drive the scaling relations for these observables. The link of low $v L_{v}(8) /$ LIR mid- to far-infrared SED shape with spatial compactness, previously indicated from local universe evidence mentioned in the introduction, is strongly supported both at low and at high redshift in the analysis of Elbaz et al. (2011).

\subsection{A Simple Model Connecting Cloud Conditions and Global SED}

Galaxy-integrated LIR is not a fundamental parameter that determines the conditions in the molecular clouds and PDRs which produce the IR emission. Rather, it has an empirical correlation with these conditions. Borrowing the terms from thermodynamics, a clear distinction should be kept between total extensive quantities which are summed over the entire galaxy (LIR, stellar, and gas masses...), and intensive quantities which are averaged, such as SSFR, SED shape, gas depletion timescale.... It is easy to imagine a galaxy in which everything is doubled (every cloud and star becomes two, etc.): extensive quantities like total LIR will be doubled, but intensive ones such as SED shape and SSFR will remain unchanged. The relations between extensive and intensive quantities will change in such a scaling. The SED shape as an intensive quantity that is related to local cloud physics should more directly scale with another intensive quantity such as the SSFR or its offset from the main sequence. The SSFR at a given redshift, which is proportional to LIR/ $M_{*}$, offers a natural scaling reference: LIR and $M_{*}$ are measurable and their ratio does not change when scaling as described.

We adopt the simplified hypothesis that main-sequence galaxies at all of our redshifts form their stars in a single type of star-forming molecular clouds (which for brevity, we will simply refer to as clouds) and discuss the implications for our findings as well as other scaling relations below in a toy model. Main-sequence galaxies with different numbers of these clouds will thus have similar mid- to far-IR SED but a different LIR. Galaxies above the main sequence will have different local cloud properties.

If the number of the clouds were proportional to the stellar mass, more stars would mean proportionally more of the same clouds and LIR emission. Our adopted Rodighiero et al. (2010) main sequence argues instead that the number of clouds scales less than proportional with the stellar mass when moving along the main sequence. This agrees with the finding in Section 3.1 
that galaxies with constant $v L_{v}(8) / \mathrm{LIR}$ are not at constant SSFR, but have an $\operatorname{SSFR}\left(M_{*}\right)$ dependency.

Comparing main-sequence galaxies of similar mass at different redshifts in this picture, we expect more of the same clouds per stellar mass at higher redshift. If similar stellar masses imply roughly similar galactic radii, then the high- $z$ galaxies have similar molecular clouds with less empty volume between them, meaning a higher surface brightness. Higher surface brightness of main-sequence galaxies is indeed observed by Wuyts et al. (2011b)

We explore the scenario of a single type of star-forming cloud in a toy model. One can define a dimensionless efficiency parameter for star formation as

$$
\frac{\dot{\rho}_{*}}{\rho_{\mathrm{Gas}}} \tau_{\mathrm{ff}}=\epsilon,
$$

where $\dot{\rho}_{*}$ is the SFR per volume element, $\rho_{\text {Gas }}$ is the gas density, and $\tau_{\mathrm{ff}}$ is the gas free-fall timescale $\left(\tau_{\mathrm{ff}} \propto \rho^{-1 / 2}\right)$ of a gravitationally bound cloud. $\epsilon$ is determined by the microphysics inside the molecular cloud and we will assume it to be a global constant. This translates to the local Kennicutt-Schmidt (KS) relation (Schmidt 1959; Kennicutt 1989, 1998b):

$$
\dot{\rho}_{*} \propto \epsilon \rho_{c}{ }^{\alpha}
$$

where we assume $\dot{\rho}_{*}$ and $\rho_{c}$ to be averages over the star-forming cloud (as opposed to the entire galaxy volume), indicated by the subscript $c$. Traditionally $\alpha=3 / 2$; however, due to various measurements deriving values in the range of $1<\alpha<1.7$ (Bouché et al. 2007; Kennicutt et al. 2007; Bigiel et al. 2008; Genzel et al. 2010) we leave this as a general parameter. The free-fall timescale is only relevant for a gravitationally bound cloud set to collapse and form stars, not for the averaged density over the entire galaxy. Hence, the above is a local relation applicable to a small-scale molecular cloud entity. We would like to integrate over the galaxy volume to derive the total, extensive measurables: SFR and mass. To do this, we define a filling factor $f$ that describes the fraction of the total volume $V$ of the galaxy which is occupied by molecular star-forming clouds. The integrated SFR over the volume $V$ is then

$$
\dot{M}_{*} \propto \epsilon \rho_{c}^{\alpha} f V
$$

We can now divide by the stellar mass $M_{*}$ and use $\rho_{c} f V=$ $\left(M_{\mathrm{Gas}} / M_{*}\right) M_{*}$ to eliminate the volume and get

$$
\frac{\dot{M}_{*}}{M_{*}} \propto\left(\frac{M_{\mathrm{Gas}}}{M_{*}}\right)\left(\epsilon \rho_{c}^{\alpha-1}\right),
$$

where $M_{\mathrm{Gas}}$ is the galaxy-integrated molecular gas mass.

The rightmost term in parentheses, which includes the average cloud density and the efficiency parameter, describes the local conditions in the star-forming regions and hence is associated with the IR SED shape. All main-sequence galaxies will have the same value in these parentheses and also the same SED. Galaxies above the main sequence will boost the $\dot{M}_{*} / M_{*}$ by changed cloud conditions, going along with SED and other changes.

From Equation (14), galaxies with the same IR SEDs will tend to have similar SSFR, modified by their gas fractions. On an SSFR versus $M_{*}$ diagram, such constant SED galaxies (indicated by constant $\nu L_{\nu}(8) / \mathrm{LIR}$ in this study) will lie on a slope which follows the slope of the $M_{\mathrm{Gas}} / M_{*}$ relation.
Comparing different redshifts, changing gas fractions (Tacconi et al. 2010) would mediate the change of main-sequence SSFR with redshift in Equation (14). This change is needed to have same cloud conditions and SED at different SSFR, but at the same SSFR offset from the redshift-dependent main-sequence $\Delta \log (\mathrm{SSFR})_{\mathrm{MS}}$.

In local galaxies, molecular gas fractions decrease gently with stellar mass, though with a large scatter (Saintonge et al. 2011a). The slope of the local main sequence (in SSFR versus $M_{*}$ ) is very similar (Brinchmann et al. 2004; Salim et al. 2007; Peng et al. 2010), in full agreement with the toy model. At higher redshifts, the dependency of $M_{\mathrm{Gas}} / M_{*}$ on $M_{*}$ is still somewhat unclear. Tacconi et al. (2010) measured the molecular gas fraction in a sample of $z \sim 1$ and $z \sim 2$ galaxies lying close to their respective main sequence. Their sample size and spread in $M_{*}$ is not yet sufficient to conclusively probe for trends of the gas mass fraction with stellar mass. Sample size also limits the conclusions of Daddi et al. (2010a) who find a near constant $M_{\mathrm{Gas}} / M_{*}$ for six $z \sim 1.5$ main-sequence galaxies.

Another way to look at Equation (14) is to divide both sides by the gas to stars mass ratio to get a dependency on the gas depletion timescale $\tau_{\mathrm{dep}}=M_{\mathrm{Gas}} / M_{*}$ instead of SSFR

$$
\tau_{\mathrm{dep}}^{-1} \propto \epsilon \rho_{c}^{\alpha-1}
$$

where $\tau_{\text {dep }}$ is an intensive quantity that is directly related to the process of star formation, unlike SSFR which involves the mass of older stars. For local galaxies, Saintonge et al. (2011b) find a relatively tight correlation between $\tau_{\text {dep }}$ and the SSFR across two orders of magnitude in SSFR: $\log \left(\tau_{\text {dep }}\right)=$ $-0.724 \log (\mathrm{SSFR})+1.54$. Interestingly, when they scale the SSFR by the change of the main sequence with redshift, the relation agrees with $z \sim 1$ and $z \sim 2$ SFGs, though the statistics are low. In Equation (15), the measurable quantity $\tau_{\mathrm{dep}}$ is equivalent to local cloud conditions and hence closely and directly related to the resulting IR SED. If indeed $\tau_{\text {dep }}$ corresponds to the resulting IR SED shape, then this supports our conclusion from Section 5 that the relation between SSFR and SED shape scales with redshift like the main sequence. At a given redshift, the decrease of $\tau_{\text {dep }}$ with increasing SSFR represents the change in molecular cloud properties as galaxies move away from the main sequence toward "bursty" compact sources.

The above suggests that the main sequence up to $z \sim 2$ is composed of galaxies with rather uniform molecular cloud properties. The difference in integrated quantities such as LIR is mostly due to the total number of clouds. For normal galaxies, dust optical depths are low at the mid- to far-infrared wavelengths studied here. Observables will thus be less sensitive to second-order effects of arrangement of these clouds than shorter wavelengths. As we gradually move away from the main sequence to higher SSFR the properties of the clouds gradually change and $\left(\epsilon \rho_{c}{ }^{\alpha-1}\right)$ increases, meaning $\tau_{\text {dep }}$ decreases and the $v L_{v}(8) / \mathrm{LIR}$ decreases, representing denser star-forming regions. In this toy model, we cannot differentiate between an increase of the average gas density of the clouds $\rho_{c}$ or the efficiency $\epsilon$.

The global KS law $\Sigma_{\text {SFR }} \propto \Sigma_{\text {Gas }}^{\alpha}$ uses the integrated molecular gas mass surface density (total $M_{\text {Gas }}$ over area) and integrated SFR surface density. When considering only main-sequence galaxies or galaxies parallel to the main sequence in general, the power expected to be retrieved is $\alpha \approx 1$ instead of the theoretical $\alpha=1.5$ or in general the $\alpha$ of the local KS law (Equation (12)), which could partially explain low $\alpha$ values quoted in recent works that distinguish between types of galaxies and atomic 
versus molecular gas masses (e.g., Gao \& Solomon 2004; Bigiel et al. 2008; Leroy et al. 2008; Genzel et al. 2010). This is because according to the above suggested model and along lines of constant $\Delta \log (\text { SSFR })_{\text {MS }}$, the increase in the surface densities is due to filling of the empty volumes by more of the same molecular clouds, increasing mean surface densities for SFR and for (molecular) gas proportionally. $\alpha=1.5$ may still apply to the individual, or even fragments of molecular clouds (local law), if it were possible to measure it on small enough scales. The various measured values for the power in the KS law highly depend on the definitions, methods, and selections used. For a discussion on the various measurements of the KS slope, see, e.g., Genzel et al. (2010).

How does Equation (14) relate to the correlation of SED shapes with LIR? This is the original way in which the CE01 library has been constructed for local galaxies and the correlation is also seen in high- $z$ galaxies (Section 7, Figure 11). As an extensive quantity, LIR must be correlated with the clouds that emit the SED through other parameters or circumstance. For example, locally, luminosities of LIR $>10^{12} L_{\odot}$ require either a very massive main-sequence galaxy which has not turned passive (rare according to the mass function), or in fact a lower mass but with a boosted SSFR due to some special event (like mergers). In such a case, LIR becomes correlated with the cloud properties because the LIR-selected sample gets increasingly dominated by galaxies above the main sequence at high LIR. The global shift to higher LIR in the relation between SED shape and LIR for $z=1-2$ galaxies (Figure 11) is in the context of the toy model's Equation (14) attributable to the increase of gas fraction with redshift for galaxies both on and above the main sequence.

How do the two correlations SED-LIR and SED- $\triangle$ SSFR $_{M S}$ fit together? A sloped main sequence produces this naturally: Over a limited mass range, a selection by LIR ( -1 slope in $\operatorname{SSFR}\left(M_{*}\right)$ ) will then produce a similar selection to one by $\triangle \mathrm{SSFR}_{\mathrm{MS}}$ from a sloped main sequence. As stated before, in the SSFR versus mass diagram we associate the slope of constant $v L_{v}(8) /$ LIR galaxies with the slope of the main sequence. Both slopes are not well determined and it is possible that this association, within the large uncertainties, is incidental. In practice, we measured $\Delta S S F R_{M S}$ from a reference line that we fixed. The more important aspect is that for the reference line we chose scales with redshift like the main sequence.

The toy model, capturing cloud properties into $\rho_{c}$ which is assumed to be identical for all main-sequence galaxies, is admittedly simplifying. Nevertheless, it is able to capture the essence of the link between SEDs and SSFR offset from the main sequence, and to motivate scalings with redshift that are related to gas content. Further insight will come from testing its assumptions, since $\rho_{c}$ may not uniquely define the kind of clouds in the galaxy and not have a 1:1 link to SED. Different cloud structures could produce a similar $\rho_{c}$, but different SED. Metallicity and nonlinear effects can also play a role, in particular the latter when the local filling factor of the molecular gas approaches $f \sim 1$ and the clouds start affecting each other. While being a sensitive tracer to the conditions in the starforming regions, $v L_{v}(8) /$ LIR can be affected by many different physical conditions of $\mathrm{PAH}$ and continuum emission, grain size distribution, and the detailed chemistry. Attenuation in the midinfrared in extreme environments, such as those encountered in local ULIRGs, can play a role in these special cases. Therefore, some scatter is to be expected in the correlation between $v L_{v}(8) / \mathrm{LIR}$ and the distance from the main sequence, LIR, gas depletion time, and other quantities.

\subsection{Comparison of $24 \mu \mathrm{m}$-based SFR Estimates Adopting Different Mid- to Far-Infrared SED Calibrations}

In Sections 5 and 7, we derived two calibrations for the $24 \mu \mathrm{m}$ photometry as an SFR indicator at redshift $\sim 2$. Both produced similar $\sim 0.4$ dex scatter in LIR(24)/LIR around $z \sim 2$ when fitting our data with the modified templates. The calibration by SSFR is applicable over a wide redshift range with implicit redshift dependency, whereas the one by LIR is redshift specific. While we have presented evidence for a smooth variation of $v L_{v}(8) /$ LIR with SSFR offset above the main sequence which we consider to be the correct physical picture, one can ask about the practical viability of other approaches.

In the following, we discuss systematic deviations as well as the scatter of derived LIR(24)/LIR for our $z \sim 2$ data, applying our relations as well as ones from the literature. We assume that only $24 \mu \mathrm{m}$ flux, redshift, and stellar mass are known. The latter is used only for methods invoking the SSFR. In the summarizing Figure 13, we look at the ratio of total LIR as derived from MIPS 24 fluxes LIR(24) to the PACs-based LIR, for the $1.5<z<2.5$ SFGs in our sample. We compare the methods of CE01, Murphy et al. (2011, M11 hereafter), Wuyts et al. (2008, W08 hereafter), Elbaz et al. (2011, E11 hereafter), and our calibrations by LIR (Equation (5)) and by $\Delta \log (\mathrm{SSFR})_{\mathrm{MS}}$ (Equation (3)). Note that the $x$-axis in this figure is the LIR as derived by PACS (labeled LIR(160) for clarity) and not LIR(24). Plotting versus LIR(24) would tend to produce positive slopes even for a perfect calibration. This is because in the presence of noise and a steeply declining luminosity function, the highest LIR(24) will tend to be dominated by upscatter of lower luminosities. In each panel, we show the results for the individual sources in our sample, the mean offset of LIR(24)/LIR, and the standard deviation around it, and finally the slope of a linear fit that is also overplotted, highlighting any systematic biases.

The intrinsic scatter in $v L_{v}(8) / \mathrm{LIR}$ within the galaxy populations seems to be significant even after binning by $\triangle \mathrm{SSFR}_{\mathrm{MS}}$ (Figure 5), as well as by LIR (Figure 10); see also Figure 3. We estimate this scatter to be about $\sim 0.2 \mathrm{dex}, 1 \sigma$, consistent with findings by Elbaz et al. (2011). For the purpose of using rest-frame $8 \mu \mathrm{m}$ for the estimation of LIR, this intrinsic scatter sets basic limits to the achievable accuracy of any of the conversions. An indirect effect of this scatter arises in calibrations for which $v L_{v}(8) /$ LIR varies steadily with LIR or with mainsequence offset, similar to our calibrations and CE01 (where $\left.\mathrm{LIR}_{\mathrm{CE} 01} \propto v L_{v}(8)^{1.5}\right)$. The scatter in the derived LIR(24) will then be nonlinearly amplified. For example, LIR(24) for a source with a higher than average $\nu L_{v}(8) / \mathrm{LIR}$ (for its LIR) will not only be overestimated proportionally to $v L_{v}(8)$, but also in addition to, because a template with lower $v L_{v}(8) /$ LIR will be applied. Finally, the scatter will also blur any systematic deficiencies in the conversion methods themselves.

The CE01 calibration (Figure 13, top left) produces a clear systematic offset for the reasons discussed throughout this paper. There is no clear trend with luminosity, and the $\sim 0.36$ dex scatter is relatively high because of the nonlinear "amplification."

Murphy et al. (2011), on the basis of mostly the data of Murphy et al. (2009), suggested an empirical correction to the LIR as estimated when using the original CE01 template library. This correction assumes that any LIR $<10^{12} L_{\odot}$ as obtained when using the CE01 templates is accurate, but applies a luminosity-dependent correction when the fit results 


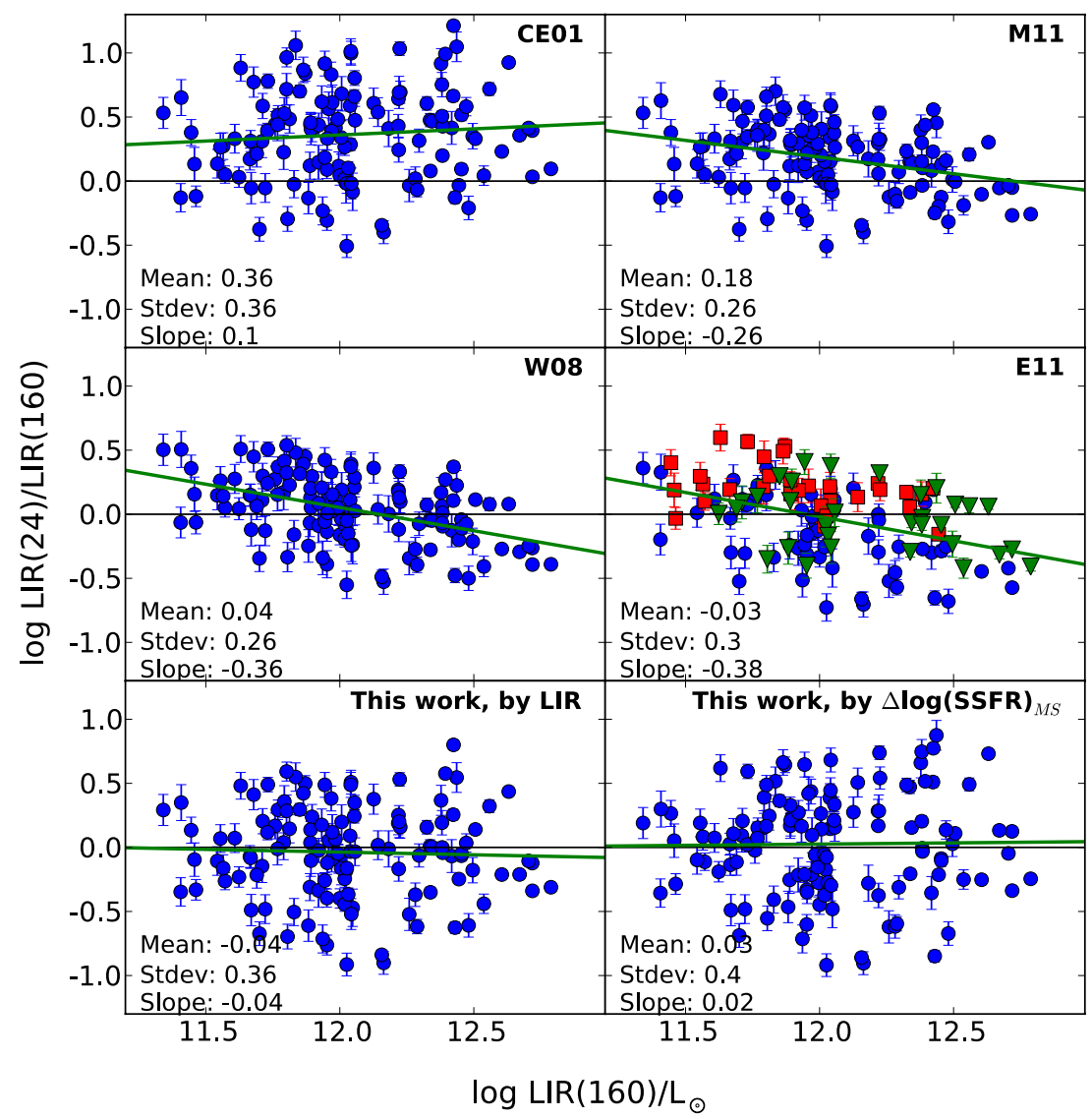

Figure 13. Comparison between various template sets and conversion methods to derive LIR from a single $24 \mu \mathrm{m}$ flux measurement. The methods correspond to Chary \& Elbaz (2001, CE01), Murphy et al. (2011, M11), Wuyts et al. (2008, W08), and Elbaz et al. (2011, E11) and the bottom panels correspond to the calibrations from Section 7 (left) and Section 5 (right). The markers and colors used in E11 correspond to the assumed template: blue circles for main sequence, red squares for starburst, green triangles for a combination of both. The green line in each panel is a simple linear trend.

(A color version of this figure is available in the online journal.)

in LIR $>10^{12} L_{\odot}$. This method does not change the templates themselves, but instead is applied to the LIR, after it is derived using the original CE01 library. This M11 correction by a power of 0.6 cancels out most of the nonlinearity built into the CE01 library, and hence also reduces the associated amplification of scatter. The calibration of M11 effectively removes the excess seen in the CE01 LIR(24) for the LIR $\sim 10^{12.5} L_{\odot}$ where it was constrained, but the luminosity of galaxies with LIR $\lesssim 10^{12} L_{\odot}$ still tends to be overestimated. It thus introduces a trend with the real LIR (Figure 13, top right). Note that the lower luminosity galaxies used by M11 to constrain their correction are at lower redshifts, plausibly explaining why systematic offsets remain for low LIR $\lesssim 10^{12} L_{\odot}$ galaxies at $z \sim 2$.

Wuyts et al. (2011a) suggested a unique luminosityindependent conversion from $24 \mu \mathrm{m}$ to LIR. The conversion is based on a template from Wuyts et al. (2008). This template has $\log \left(v L_{v}(8) / \mathrm{LIR}\right)=-0.92$ with the MIPS24 filter at $z=$ 2. This is also the median value of the GOODS-S sample used in this work. This correction produces for our sample a very good overall correction (small mean LIR(24) excess), but with a clear overall trend with LIR. The luminosities derived by this conversion method are overestimated at low LIR and underestimated at high LIR (Figure 13, middle left). The scatter is low because intrinsic scatter in $v L_{v}(8) /$ LIR is not amplified by the method-errors and real variation are propagated linearly. Given our results, both such a residual trend and a moderate scatter will be a feature of correction recipes that are adopting only a single template. In principle, such a single $v L_{v}(8) / \mathrm{LIR}$ conversion is in contradiction to the clear LIR or SSFR dependence that we find in this study. In practice, the accuracy of such a conversion depends on the selection of the sample to which it is applied, and will develop biases when selecting mainly low-luminosity (main-sequence) galaxies or very luminous galaxies (high above the main sequence). Since it encodes neither main-sequence offset nor a redshift dependence, it can also create redshift-dependent biases. Main-sequence normalization and flux limit of many surveys scale with redshift in a roughly similar way, however. This will reduce such effects in practice.

Figure 6 suggests that a replacement of the continuous SED calibration with SSFR by two discrete values for sources on and sources above the main sequence (the approach taken by Elbaz et al. 2011) can potentially perform well as a two-step approximation to the trend. The change in $v L_{v}(8) / \mathrm{LIR}$ between main sequence and sources with a $\sim 0.5$ dex higher SSFR is similar to our $\sim 0.4$ dex scatter around the calibration. Such a treatment may be particularly applicable if comparing samples on the main sequence with merger-dominated samples and would directly correspond to the two modes of star formation seen in CO-based studies of the Kennicutt-Schmitt law (Genzel et al. 2010; Daddi et al. 2010b). While we do not find such a corresponding bi-modality in the $v L_{v}(8) / \mathrm{LIR}$ relation, it is beyond the scope of this work to study to which extent the smooth trend in Figure 6 is due to smooth changes of conditions 
within individual galaxies versus a varying mix of "normal" galaxies and "mergers" in a given SSFR bin.

The middle right panel of Figure 13 shows the result of applying the E11 method to our sample. We have adopted here the main-sequence parameterization of E11 (mass independent in SSFR) and their approach of separating main sequence and "starbursts" at SSFR a factor of two above the main sequence. The two templates in E11 were in practice derived by separating the galaxies in values equivalent to $v L_{v}(8) / \mathrm{LIR}$ and not strictly in SSFR. Without prior knowledge on the SFR in the galaxy, the selection of the correct template can sometimes be ambiguous, with permitted solutions for both templates at a given $24 \mu \mathrm{m}$ flux and redshift. The $\operatorname{LIR}(24)$ difference then is $\sim 0.25 \mathrm{dex}$ between the two. These cases are marked as green triangles in Figure 13 and we adopted the mean LIR from the two templates. The application of the E11 method to our sample removes the bias and shows low scatter, but leaves a residual slope. In these properties, practical results from this two-template SSFR-based approach resemble the single W08 template method.

The template calibrations derived in this work produce negligible trends and biases with LIR and $\Delta \log (\mathrm{SSFR})_{\mathrm{MS}}$, but a scatter which is 1.2-1.5 times larger than the other methods (Figure 13, bottom). Much of the increase in scatter is related to the nonlinearity in the conversion from $8 \mu \mathrm{m}$ flux to LIR. This nonlinearity in CE01, which also prevails in our calibrations and is represented by the strong negative slopes seen in Figure 11, means that a scatter in $v L_{v}(8)$ is super-linearly enhanced by a power of $\sim 1.5$ when converting to LIR. With respect to a single template, the improvement by a more detailed $v L_{v}(8) /$ LIR versus LIR relation removes bias, but is too small to compensate for scatter in $v L_{v}(8)$ and its nonlinear increase in the conversion to LIR. When using the template calibrations by $\Delta \log (\mathrm{SSFR})_{\text {MS }}$ (Figure 6, Equation (3)), the nonlinearity is even higher, with a power of $\sim 2$. Deviations in $v L_{v}(8)$ can be due to the photometric errors and due to the intrinsic scatter in the population. In our sample both have a significant contribution to the overall scatter. When requiring knowledge of the masses (as when using $\left.\Delta \log (\mathrm{SSFR})_{\mathrm{MS}}\right), \sim 0.2$ dex errors in the estimated masses are an additional source of variation. Our $\Delta \log (\mathrm{SSFR})_{\mathrm{MS}}$ calibration introduces a higher degree of nonlinearity, which increases the other sources of variation by larger factors than for other methods. This makes the findings from Section 3.1 of best correlations with $\Delta \log (\mathrm{SSFR})_{\mathrm{MS}}$, consistent with the larger scatter in LIR(24)/LIR reported here.

Perhaps surprisingly, for $24 \mu \mathrm{m}$-based LIR derivations a simplified linear conversion will often produce less scatter in the resulting luminosities than when using LIR or $\Delta \log (\mathrm{SSFR})_{\mathrm{MS}}$ dependent templates, even though the latter methods better describe the true relation and remove bias. The difference between the methods, and in particular between linear and nonlinear methods, will vary depending on the sample selection and the various sources of noise (photometry, redshifts, and masses). Any attempt to correct for the sloped trends seen in Figure 13 will inevitably include a nonlinear scaling that will trade reduced bias for increased scatter.

\section{CONCLUSIONS}

We have obtained deep 70, 100, and $160 \mu \mathrm{m}$ Herschel-PACS photometric maps of the GOODS fields as part of the PEP project. Using this data, we study the relation between $8 \mu \mathrm{m}$ rest-frame emission and the total IR luminosity $v L_{v}(8) / \mathrm{LIR}$ of $z \sim 1$ and $z \sim 2$ galaxies. The deep far-infrared observations allow us for the first time to reliably measure the total infrared luminosities of normal SFGs on the $z \sim 2$ main sequence with little need of extrapolation or stacking. We studied the typical SED shapes by binning the galaxies according to their $\triangle \mathrm{SSFR}_{\mathrm{MS}}$ distance from the main sequence, as well as by LIR. We found the SED shape that best describes the population using a photometric mean SED fitting method (Section 4). For a subsample of 16 sources at $z \sim 2$ and 9 at $z \sim 1$, we tested the results from the photometric fit against stacked midinfrared spectra and found a good match between the corrected templates and the spectra in the range 6-12 $\mu \mathrm{m}$. Finally, we examined a sample of X-ray AGNs which we compared to the SFG population.

Our main findings are summarized below.

1. The excess in $8 \mu \mathrm{m}$ emission with respect to LIR for galaxies at $z>1.5$, reported in earlier studies, can be attributed almost entirely to enhanced emission of PAHs with respect to the locally calibrated templates that were used. This is verified using a combination of LIR from Herschel-PACS and stacked deep Spitzer-IRS spectroscopy. No continuum component from an obscured AGN is typically required to explain the mid-infrared emission of far-infrared bright SFGs with $\log \left(\mathrm{LIR} / L_{\odot}\right) \gtrsim 11.5$. A similar effect can be observed with a $16 \mu \mathrm{m}$ filter at $z \sim 1$ (8 $\mu \mathrm{m}$ rest frame).

2. $v L_{v}(8) /$ LIR correlates with both the absolute LIR and the $\triangle \mathrm{SSFR}_{\mathrm{MS}}$ distance from the main sequence of SFGs. The latter gives a tighter correlation without explicit redshift dependence (see also Elbaz et al. 2011). We derive calibrations for both relations which we implement on the CE01 template library as an example. $24 \mu \mathrm{m}$ can then be used to estimate LIR in $0.7<z<2.5$ galaxies using the rescaled templates. The scatter is about a factor of 2.5 in our sample, but with no bias or trends with luminosity. Calibrating the templates by LIR, $z \sim 1$ and $z \sim 2$ galaxies have SEDs similar to local galaxies with $\log (\mathrm{LIR})$ lower by $0.5 \mathrm{dex}$ and 0.8 dex, respectively.

3. The correlation of the SED shape with the distance from the redshift-dependent main sequence suggests that starforming molecular clouds in main-sequence galaxies have similar physical conditions at all redshifts $z<2.5$, with only their number and filling factor changing along the main sequence and between redshifts. On the main sequence, $\log \left(v L_{v}(8) / \mathrm{LIR}\right)=-0.58$ when the $8 \mu \mathrm{m}$ flux is measured through the MIPS $24 \mu \mathrm{m}$ filter at $z=2$. For higher SSFR above the main sequence, $v L_{v}(8) /$ LIR decreases with increasing $\triangle \mathrm{SSFR}_{\mathrm{MS}}$. This can be interpreted as more compact star formation, as suggested by Elbaz et al. (2011), in a way consistent with scaling trends observed for other ISM emissions.

4. The majority of X-ray AGNs in our sample have midinfrared emission which is completely dominated by the star formation related emission (mainly PAHs). This is also seen in a stacked IRS spectrum of four X-ray AGNs. Only one other, the brightest AGN, shows a significant midinfrared excess (factor $~ 3.6$ ) both in photometry and the IRS spectrum, with respect to the (corrected) template of a SFG. We derive a condition on the AGN luminosity and LIR in order for the AGN to contribute an even or greater $8 \mu \mathrm{m}$ emission than the star formation. The required AGN luminosities for massive $z \sim 2$ main-sequence galaxies with $\log \left(\mathrm{LIR} / L_{\odot}\right) \sim 12$ are high $\left(L_{\mathrm{AGN}} \gtrsim 10^{45.8} \mathrm{erg} \mathrm{s}^{-1}\right)$, which makes galaxies with a true $8 \mu \mathrm{m}$ excess due to the AGN component rare. The fraction of AGNs dominating 
the $8 \mu \mathrm{m}$ emission is strongly dependent on the selection and is likely to increase for $\log \left(\mathrm{LIR} / L_{\odot}\right) \lesssim 11.3$.

PACS has been developed by a consortium of institutes led by MPE (Germany) and including UVIE (Austria); KUL, CSL, IMEC (Belgium); CEA, OAMP (France); MPIA (Germany); IFSI, OAP/OAT, OAA/CAISMI, LENS, SISSA (Italy); and IAC (Spain). This development has been supported by the funding agencies BMVIT (Austria), ESA-PRODEX (Belgium), CEA/CNES (France), DLR (Germany), ASI (Italy), and CICYT/MCYT (Spain).

\section{APPENDIX A \\ DETERMINING LIR}

The first and most important piece of information we need for each galaxy is its 8-1000 $\mu \mathrm{m}$ LIR. As discussed in Section 3, the observed $24 \mu \mathrm{m}$ flux is problematic as an LIR estimator for $z \sim 2$ galaxies and its deviation from the local templates is one of the main issues we wish to investigate. We therefore exclude it from the LIR determination which we base exclusively on the energetically dominant rest-frame far-infrared data. This is done also to avoid subtle bias by which, depending on the specific adopted weighting scheme, $24 \mu \mathrm{m}$ fluxes might affect the LIR from template fits to combined mid- and farinfrared data. Only a small number of very luminous galaxies are detected at $70 \mu \mathrm{m}$ and only GOODS-S was observed at this wavelength with PACS, which leaves us with PACS $160 \mu \mathrm{m}$ and possibly PACS $100 \mu \mathrm{m}$ to constrain both shape and scale of the SEDs.

Our basic strategy is to derive LIR from fitting CE01 templates to PACS photometry points close to rest-frame $60 \mu \mathrm{m}$. For reasons described below, at $z \sim 2$ we use only the observed $160 \mu \mathrm{m}$ and fit only the scale, maintaining the CE01 LIR-SED shape relation. At $z \sim 1$, we use both 100 and $160 \mu \mathrm{m}$ and fit both SED shape and scale. We sometimes designate the PACS-based LIR at $z \sim 2$ as LIR(160) for a clear distinction from a MIPS-based LIR(24), but refer to 8-1000 $\mu \mathrm{m}$ rest wavelength in all cases.

For the higher redshift $z \sim 2$ sources, detection rates are limited in the 100 and $70 \mu \mathrm{m}$ bands compared to $160 \mu \mathrm{m}$ for most luminosity bins. Selecting only the sources with two far-infrared points will bias our sample to the galaxies with bluer far-infrared colors and limit the ability to use stacking to compensate for the non-detections.

Fortunately, with Herschel-PACS we are measuring LIR close to the far-infrared peak if using PACS $160 \mu \mathrm{m}$ at $z \sim 2$. The determination of LIR is thus much less sensitive to the exact shape of the assumed SED. The flux density at the rest-frame wavelength of $60 \mu \mathrm{m}$ is a particularly good measurement for a monochromatic luminosity determination as we shall explain below.

Elbaz et al. (2010) demonstrated that for redshifts $1<z<3$, fitting the Chary \& Elbaz (2001) SED library to a single PACS $160 \mu \mathrm{m}$ measurement results in an excellent agreement with the LIR measured from combined Herschel PACS+SPIRE photometry (sampling the full far-infrared SED peak). The robustness of LIR determined from monochromatic $160 \mu \mathrm{m}$ luminosity can be understood by looking at the templates normalized to their total IR luminosity. The conversion factor from the observed flux $F_{v}(\lambda)$ to the total IR luminosity is $\nu L_{v}(\lambda) /$ LIR. Several templates from the CE01 SED library are plotted in Figure 14 in the $v L_{v}(\lambda) /$ LIR scale. The templates

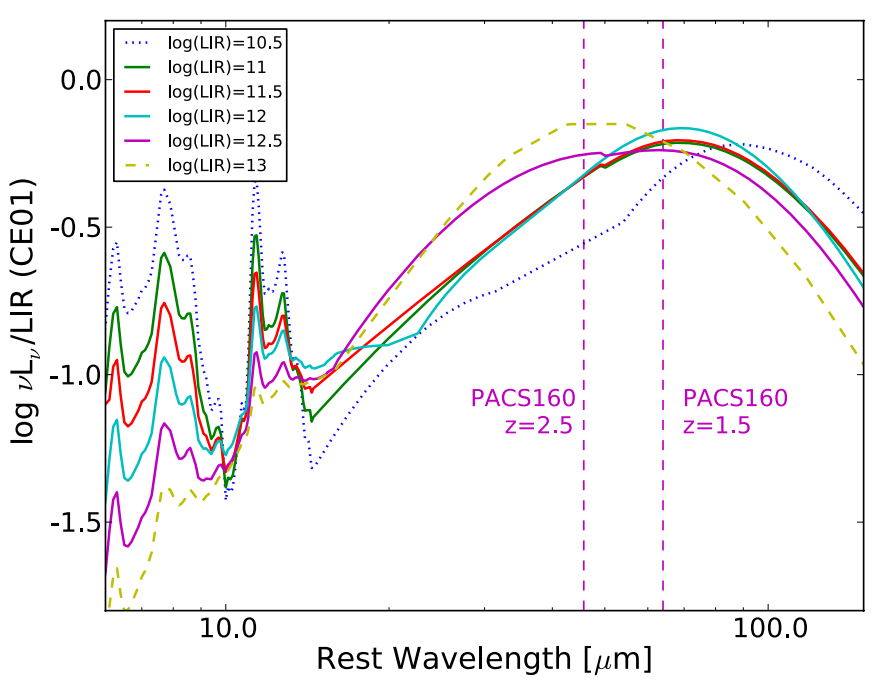

Figure 14. $v L_{v} /$ LIR for various templates of relevant luminosities from the CE01 SED library. Vertical dashed lines indicate the rest-frame wavelengths observed at $160 \mu \mathrm{m}$ for $z=1.5$ and 2.5.

(A color version of this figure is available in the online journal.)

for the relevant luminosities of LIR $=10^{11}-10^{13} L_{\odot}$ cross each other between 50 and $60 \mu \mathrm{m}$, where the $160 \mu \mathrm{m}$ filter samples the SED at $1.5<z<2.5$ (dashed vertical lines). Of course, at low enough luminosities, such as $\log \left(\mathrm{LIR} / L_{\odot}\right)=10.5$ $\left(\sim 3.5 M_{\odot} \mathrm{yr}^{-1}\right)$, the template deviates for the typical $v L_{v} / \mathrm{LIR}$ at $60 \mu \mathrm{m}$. However, our typical luminosities are more than an order of magnitude higher than that and such cold SEDs will not be common.

Nearly identical $v L_{v}(\lambda) /$ LIR values for the different templates at a given wavelength mean that for the redshifts at which the filter is centered on this wavelength, LIR can be measured regardless of the specific template that is selected. Possible systematic errors due to selecting a template different from the optimal one are very small and can be visually seen in Figure 14 as the distance between the SED lines (up to $0.1 \mathrm{dex}$ around $60 \mu \mathrm{m}$ ). This fortunate coincidence around $60 \mu \mathrm{m}$ will gradually worsen if the dust temperatures get significantly lower than the local LIR- $T$ relation implemented in the CE01 library. Hwang et al. (2010) studied deep Herschel data from the farinfrared to submillimeter and report that this is not the case and this result agrees with the accuracy of LIR measured from $160 \mu \mathrm{m}$ as found by Elbaz et al. (2010). Further support is given to this by the deeper data of Elbaz et al. (2011). We therefore measure LIR for $z \sim 2$ galaxies by fitting CE01 templates to $160 \mu \mathrm{m}$ fluxes alone, even in cases where shorter wavelength fluxes are available.

The reason not to use PACS 70 and $100 \mu \mathrm{m}$ even when available is twofold: one is that the fairly low detection rates at these wavelengths. If we use the added data points per galaxy to relax the luminosity-template relation in the library it means that we will be treating the part of the population which is detected at $100 \mu \mathrm{m}$ differently than the non-detections. The other is that the $z \sim 270$ and $100 \mu \mathrm{m}$ bands observe $\sim 25$ and $35 \mu \mathrm{m}$ rest, far from the SED peak and a region of the SED which is not part of the modified blackbody emission and for which the $v L_{v}(\lambda) /$ LIR values can vary significantly between templates (Figure 14). Using this SED region to constrain LIR means that we allow a measurement that represents a small fraction of the total luminosity to determine LIR. This can lead to systematic errors if the selected template is inaccurate and 


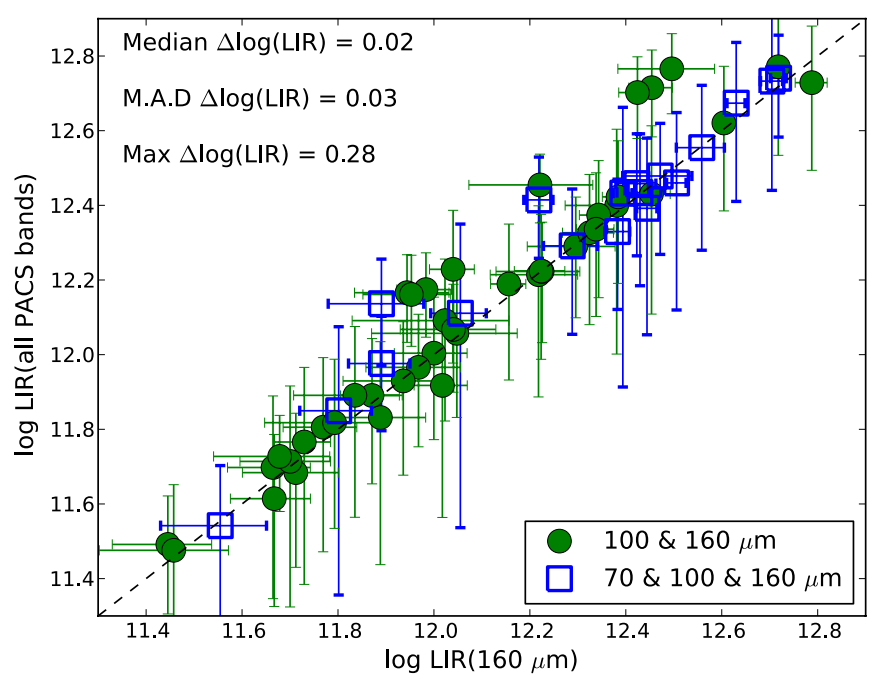

Figure 15. Comparison of total infrared luminosity LIR(all PACS bands) derived from all available PACS bands (at least two detections) vs. total infrared luminosity LIR(160) from $160 \mu \mathrm{m}$ alone. LIR(all PACS bands) is determined by fitting all available PACS bands with free scale and template shapes in the CE01 library. LIR(160), used throughout the paper, is determined by fitting $160 \mu \mathrm{m}$ alone, maintaining the original luminosity-template relation in the library. We select all $1.5<z<2.5$ galaxies in our sample that have two or three PACS fluxes (45 and 15 out of 128). The statistics for $\Delta \log (\mathrm{LIR})=\log (\mathrm{LIR}($ all PACS bands $))-\log (\operatorname{LIR}(160))$ are given in the figure. Error bars represent formal random errors only, propagated from the photometric errors.

(A color version of this figure is available in the online journal.)

to even larger errors if we do not thaw the luminosity-template relation in the library. The combination of these two effects makes the potential biases and systematic errors outweigh the gain in added constraints to the LIR determination.

In any case, the difference when including or excluding the shorter wavelengths is quite small as we demonstrate in Figure 15. In our $z \sim 2$ sample, considering only sources that have $160 \mu \mathrm{m}$ and at least one other PACS band detections (half of our full $z \sim 2$ sample), we fit all available PACS bands with CE01 templates (free template and scales, $\chi^{2}$ minimization) and compare with the LIR derived by a monochromatic $160 \mu \mathrm{m}$ fit. The median difference and MAD between the two methods are 0.02 and 0.03 dex and the maximum difference is $0.28 \mathrm{dex}$. The added (and possibly biased) constraint provided by the 100 and $70 \mu \mathrm{m}$ points is used for selecting a slightly different template, that still results in nearly the same LIR which is determined by the $160 \mu \mathrm{m}$ flux almost independently (see Figure 14). Only 5 of 60 galaxies deviate in their monochromatic LIR(160) from the multi-band LIR(PACS) by more than $1 \sigma$, the largest of which is $1.5 \sigma$. One should keep in mind that due to the detection limits, many galaxies with real $100 / 160 \mu \mathrm{m}$ flux ratios that are lower than our sample values will simply not be detected in $100 \mu \mathrm{m}$ and are not represented in this comparison.

Concerning our lower redshift $z \sim 1$ bin, the far-infrared rest-frame wavelengths scanned by the three PACS photometer bands for $0.7<z<1.3$ spans across 20-95 $\mu \mathrm{m}$, overlapping in large part the range covered for $1.5<z<2.5(20-65 \mu \mathrm{m})$. At these lower redshifts, the PACS $160 \mu \mathrm{m}$ filter has moved farther away from the rest-frame $60 \mu \mathrm{m}$ and the PACS $100 \mu \mathrm{m}$ filter has moved closer. The lowest luminosities in the lower redshift sample are of $\log \left(\mathrm{LIR} / L_{\odot}\right) \approx 10.8$, which may include colder SEDs than those for which the $60 \mu \mathrm{m}$-derived LIR is ideal. By coincidence, for the lower luminosities of the $z \sim 1$ sample $\left(\log \left(\mathrm{LIR} / L_{\odot}\right) \lesssim 12\right)$, the templates cross at rest $80-90 \mu \mathrm{m}$
(Figure 14), which is again the rest wavelength of the $160 \mu \mathrm{m}$ filter. At these redshifts $100 \mu \mathrm{m}$ has a much higher detection rate (100\% of the $160 \mu \mathrm{m}$ sources in most bins we use) and probes a much more useful rest-frame wavelength $(\sim 50 \mu \mathrm{m})$ than it does at $z \sim 2$. The arguments for using only $160 \mu \mathrm{m}$ mentioned above do not apply to this sample. For LIR determination at $z \sim 1$, we fit the best combination of CE01 template shape and scale (as two free parameters) to the 100 and $160 \mu \mathrm{m}$ points simultaneously. For a few cases at the lowest luminosities where only $160 \mu \mathrm{m}$ is available, a monochromatic fit to LIR is used.

\section{APPENDIX B}

\section{STACKING ANALYSIS}

\section{B.1. Measuring the Stacked Flux}

Sources that are undetected in 70 or $100 \mu \mathrm{m}$ (but detected at $160 \mu \mathrm{m})$ are stacked to produce a mean photometric point representing this population. Stacking is performed into a residual map from which all individually detected sources have been removed. This is done in order to minimize the contamination from much brighter nearby sources. The viable depth of stacks is limited by the density of neighboring sources which are brighter than the typical sources in the stack. In this work, we stack into the 70 and $100 \mu \mathrm{m}$ images on the position of MIPS $24 \mu \mathrm{m}$ sources, which are detected at $160 \mu \mathrm{m}$. This means that the brightness of our stacked sources is likely just below the detection threshold, or they will not have been detected at $160 \mu \mathrm{m}$. By removing the individual detections, our sources of interest are among the brightest sources that are still left in the image.

The background level for each stacked image is estimated from the position of the peak in the pixel value distribution $P(D)$. This method is more reliable than measuring the background from the stacked image itself. Due to selection effects, prior positions will tend to be at a minimum distance from the neighboring sources, thus the priors tend to be positioned at local minima in the map, which is reflected as a depressed background level at the center of the stacked image. Background estimated from the edges of the stacked image will tend to be higher than the background at the position of the stacked source. Subtracting the background according to the $P(D)$ peak and extracting the flux using a limited-radius PSF avoid this issue.

\section{B.2. Stacked $v L_{v} /$ LIR}

The value of interest for the stack is $\nu L_{\nu}\left(\lambda_{0}\right) / \mathrm{LIR}$, at a certain rest-frame wavelength $\lambda_{0}$. When converting from stacked flux to $v L_{v}\left(\lambda_{0}\right) / \mathrm{LIR}$, one must account for the different redshifts of the stacked sources (affecting both the luminosity distance and the rest wavelength), as well as for the different (known) individual LIR. We use the following weighted mean to get the stack mean $v L_{v} / \mathrm{LIR}$ :

$$
\lambda_{0} \equiv \lambda_{\text {filter }} /(1+\bar{z})
$$

$v L_{v}\left(\lambda_{0}\right) /\left.\mathrm{LIR}\right|_{\text {stack }}=N_{\text {stack }} \cdot F_{v}\left(\lambda_{\text {filt }}\right)\left[\sum_{i=1}^{N_{\text {stack }}} \frac{\lambda_{\text {filt }}\left(\frac{1+z_{i}}{1+\bar{z}}\right)^{\alpha} \mathrm{LIR}_{i}}{c 4 \pi D_{i}^{2}}\right]^{-1}$,

where $\lambda_{\text {filt }}$ is the filter central wavelength and $\lambda_{0}$ is the adopted rest-frame wavelength for the stacked flux. $\bar{z}$ and $z_{i}$ are the mean and source- $i$ redshifts, $N_{\text {stack }}$ is the number of sources in the stack, $F_{v}$ is the stacked observed flux, and $D_{i}$ is the luminosity distance for each source. $\alpha$ is the approximate slope 
of the SED $\left(v L_{v} \propto \lambda^{\alpha}\right)$ at $\lambda_{0}$, which is used to correct the fluxes to a common $\lambda_{0}$. The exact value of $\alpha$ makes only a small difference in the limited redshift intervals we use-we assume $\alpha \approx 1.5$ close to the slope of the LIRG templates in CE01, which is justified posteriorly.

\section{B.3. Uncertainty on the Stacked Flux}

There are two kinds of uncertainties regarding the stacked flux as an estimator to the mean flux of the population: one is photometric, i.e., the significance of the detection of a source in the stacked image. The other is on the stacked flux as the estimator to the true mean flux of the stacked population as derived from a finite size sample. The latter depends on the flux distribution within the individual sources in the stack.

In order to estimate the photometric uncertainty $\sigma_{\text {phot }}$, we create a large number of additional stacks, each using $N$ random positions in the image, where $N$ is the number of stacked sources in the real stack. Each random stack is treated in the same way the real stack and a source flux is measured. The standard deviation in the fluxes measured from the random stacks is taken as the absolute flux uncertainty. This determines the stacked-source detection significance, i.e., the probability to produce such a flux measurement from a completely meaningless stack. For stacks without a detected source (flux $<3 \sigma_{\text {phot }}$ ), a flux of $3 \sigma_{\text {phot }}$ is taken as the upper limit.

The uncertainty on the stacked flux as an estimator to the mean source flux of the stacked population is estimated using a bootstrap method: from the list of priors in the stack a new list, identical in size, is created by random re-sampling with replacements. We perform a large number of these resamplings and produce a distribution of measured fluxes. A $68 \%$ confidence interval is calculated from this distribution. The bootstrap error on the mean stacked flux of large stacks already includes photometric flux errors. However, on a small stacked sample they might be underestimated. Therefore, the larger of the two is taken as the final uncertainty.

\section{APPENDIX C \\ THE MEAN SED REDSHIFT SCAN METHOD: $\chi^{2}$ MINIMIZATION}

When fitting an SED template to the collection of photometric points from the subsample and applying a $\chi^{2}$ minimization, one must take into account that these measurements are not repeated measurements of the same source. The measurements scatter not only due to the errors related to the photometry of each measurement $\sigma_{\text {phot }}$, but also due to the intrinsic scatter in the population $\sigma_{\text {pop }}$. Neglecting to account for this will result in a biased fit which is dominated by the few points with the best S/ $\mathrm{N}$; these would be the galaxies with the bluer far-infrared colors that are better detected at 70 and $100 \mu \mathrm{m}$ than the redder ones. $\sigma_{\text {pop }}$, the intrinsic scatter in the $v L_{v}\left(\lambda_{0}\right) /$ LIR of the population, must be estimated and included in the standard deviation for each data point when calculating the $\chi^{2}$ to be minimized:

$$
\sigma_{\nu L_{v} / \mathrm{LIR}}^{2}=\sigma_{\text {phot }}^{2}+\sigma_{\text {pop }}^{2}
$$

The inclusion of $\sigma_{\text {pop }}$ has an important implication: for cases in which $\sigma_{\text {pop }} \gtrsim \sigma_{\text {phot }}$, measurements of different sources in a given filter have nearly the same weight regardless of the photometric error. We must emphasize again that this is the appropriate weighting when doing a repeated sampling of a population rather then repeated measurements of the same object.
When estimating the intrinsic $v L_{v} /$ LIR scatter in a population of galaxies, two things need to be taken into account: one is that the photometric points from different sources are for different rest-frame wavelengths and need to be $k$-corrected to a common wavelength. The other is that for most cases, a significant fraction of the sources are not detected individually and are included as a stacked mean instead.

When the significant majority of the sources (more than $80 \%$ ) are detected by the given filter, we can ignore the small fraction of undetected sources and estimate the scatter from the detections only. For this purpose, we correct all $v L_{v} /$ LIR of the same filter to a common rest-frame wavelength (assuming a $\nu L_{v} \propto \lambda^{1.5}$ approximated slope) and calculate the standard deviation in $v L_{v} /$ LIR. When binning the galaxies by deviation from the main sequence, $\sigma_{\text {pop }}$ is also estimated from the detections only: in a selection by constant SSFR, the fainter, non-detected sources tend to be those of galaxies of lower absolute luminosity, not necessarily of lower $v L_{v} / \mathrm{LIR}$. Therefore, detections and non-detections are mixed in $v L_{v} /$ LIR of the far-infrared filters. While a weak trend with luminosity does exist, it is a secondary effect for $\sigma_{\text {pop }}$ estimation.

When selecting by LIR and a significant fraction (over 20\%) of the population is individually undetected in the given filter, the scatter is unresolved in its lower part and the weighted mean of the distribution is yet to be determined. In order to estimate the standard deviation of the full population, we approximate the distribution to be normal. In this case, we can use the fact that in a narrow LIR bin the detected and non-detected source populations are fairly well separated by the detection threshold. For the stacked sources, we only know their mean $v L_{v}\left(\lambda_{0}\right) /$ LIR and we calculate the same value for the detections using Equation (B2). We then find the standard deviation of a normal distribution, which when split into two subpopulations at a value $x_{c}$, the sizes of the two subpopulations $\left(x<x_{c}\right.$ and $x>x_{c}$ ) have the same ratio as $N_{\text {detect }} / N_{\text {stack }}$ and the difference between their means match the difference in the means of the detections and non-detections $\left(\mu_{\text {detect }}-\mu_{\text {stack }}\right)$. This problem has a simple and easy numerical solution.

\section{APPENDIX D}

\section{CALIBRATING A TEMPLATE LIBRARY BY $\delta \log (\mathrm{SSFR})_{\mathrm{MS}}$}

In this appendix, we describe how to apply the calibration derived in Section 5 to a template library, expressed as a function of $v L_{v}(8) / \mathrm{LIR}$ or LIR, which can then be fitted to a galaxy with a known mass and a redshift. The additional implicit information we need is the redshiftdependent main-sequence $\operatorname{SFR}\left(M_{*}, z\right)_{\text {MS }}$, which was defined in Section 4 and is based on the calibration of Rodighiero et al. (2010). The distance from the main sequence is then $\Delta \log (\mathrm{SSFR})_{\mathrm{MS}}=\log \left(\mathrm{SFR} / M_{*}\right)-\log \left(\mathrm{SFR}\left(M_{*}, z\right)_{\mathrm{MS}} / M_{*}\right) . \mathrm{We}$ can use either Equation (3) or Equation (4) to eliminate $\Delta \log (\mathrm{SSFR})_{\mathrm{MS}}$ and find a direct association between a template defining property $\left(v L_{v}(8) / \mathrm{LIR}\right.$ or $\left.\Lambda_{\mathrm{CE} 01}\right)$ and the LIR it should be scaled to. We will proceed with the specific example of the CE01 library and define $\chi$ as the ratio LIR/SFR (Equation (1)):

$$
\begin{aligned}
\log \left(\frac{\mathrm{LIR}}{L_{\odot}} \cdot \chi\right)= & \frac{\log \left(\Lambda_{\mathrm{CE} 01} / L_{\odot}\right)-10.58}{1.44} \\
& +\log \left(\frac{\operatorname{SFR}\left(M_{*}, z\right)_{\mathrm{MS}}}{M_{\odot} \mathrm{yr}^{-1}}\right)
\end{aligned}
$$


Thus, we scale each of the $\log \left(\Lambda_{\mathrm{CE} 01} / L_{\odot}\right)>10.38$ templates in the original library to the new LIR, simply by applying a factor LIR $/ \Lambda_{\text {CE01 }}$ to the template. The $\log \left(\Lambda_{\mathrm{CE} 01} / L_{\odot}\right)<10.38$ templates in the library either have $v L_{v}(8) / \mathrm{LIR}>-0.58$ or quickly become degenerate in this value. Our calibration does not deal with these low-luminosity templates, which tend to represent galaxies well below the current IR detection limits at the redshifts of interest.

\section{REFERENCES}

Alexander, D. M., Bauer, F. E., Brandt, W. N., et al. 2003, AJ, 126, 539 Alexander, D. M., Bauer, F. E., Chapman, S. C., et al. 2005, ApJ, 632, 736 Balestra, I., Mainieri, V., Popesso, P., et al. 2010, A\&A, 512, A12 Barger, A. J., Cowie, L. L., \& Wang, W. 2008, ApJ, 689, 687 Bauer, F. E., Alexander, D. M., Brandt, W. N., et al. 2004, AJ, 128, 2048 Bauer, F. E., Yan, L., Sajina, A., \& Alexander, D. M. 2010, ApJ, 710, 212 Berta, S., Magnelli, B., Nordon, R., et al. 2011, A\&A, 532, 49 Bigiel, F., Leroy, A., Walter, F., et al. 2008, AJ, 136, 2846 Bouché, N., Cresci, G., Davies, R., et al. 2007, ApJ, 671, 303 Bouché, N., Dekel, A., Genzel, R., et al. 2010, ApJ, 718, 1001 Brammer, G. B., van Dokkum, P. G., \& Coppi, P. 2008, ApJ, 686, 1503 Bressan, A., Panuzzo, P., Buson, L., et al. 2006, ApJ, 639, L55 Brinchmann, J., Charlot, S., White, S. D. M., et al. 2004, MNRAS, 351, 1151 Capak, P., Cowie, L. L., Hu, E. M., et al. 2004, AJ, 127, 180 Chabrier, G. 2003, ApJ, 586, L133

Chanial, P., Flores, H., Guiderdoni, B., et al. 2007, A\&A, 462, 81 Chapman, S. C., Blain, A. W., Smail, I., \& Ivison, R. J. 2005, ApJ, 622, 772 Chary, R., \& Elbaz, D. 2001, ApJ, 556, 562

Condon, J. J., Huang, Z., Yin, Q. F., \& Thuan, T. X. 1991, ApJ, 378, 65 Contursi, A., Kaufman, M. J., Helou, G., et al. 2002, AJ, 124, 751 Daddi, E., Alexander, D. M., Dickinson, M., et al. 2007a, ApJ, 670, 173 Daddi, E., Bournaud, F., Walter, F., et al. 2010a, ApJ, 713, 686 Daddi, E., Dickinson, M., Morrison, G., et al. 2007b, ApJ, 670, 156 Daddi, E., Elbaz, D., Walter, F., et al. 2010b, ApJ, 714, L118 Dale, D. A., Helou, G., Contursi, A., Silbermann, N. A., \& Kolhatkar, S. 2001, ApJ, 549, 215

Damen, M., Förster Schreiber, N. M., Franx, M., et al. 2009, ApJ, 705, 617

Dasyra, K. M., Tacconi, L. J., Davies, R. I., et al. 2006, ApJ, 651, 835

Desai, V., Armus, L., Soifer, B. T., et al. 2006, ApJ, 641, 133

Desert, F., Boulanger, F., \& Puget, J. L. 1990, A\&A, 237, 215

Downes, D., \& Solomon, P. M. 1998, ApJ, 507, 615

Dunne, L., Ivison, R. J., Maddox, S., et al. 2009, MNRAS, 394, 3

Elbaz, D., Daddi, E., Le Borgne, D., et al. 2007, A\&A, 468, 33

Elbaz, D., Dickinson, M., Hwang, H. S., et al. 2011, A\&A, 533, 119

Elbaz, D., Hwang, H. S., Magnelli, B., et al. 2010, A\&A, 518, L29

Erb, D. K., Steidel, C. C., Shapley, A. E., et al. 2006, ApJ, 647, 128

Fadda, D., Yan, L., Lagache, G., et al. 2010, ApJ, 719, 425

Fadely, R., Allam, S. S., Baker, A. J., et al. 2010, ApJ, 723, 729

Fontana, A., Pozzetti, L., Donnarumma, I., et al. 2004, A\&A, 424, 23

Fontana, A., Salimbeni, S., Grazian, A., et al. 2006, A\&A, 459, 745

Förster Schreiber, N. M., Genzel, R., Bouché, N., et al. 2009, ApJ, 706, 1364

Förster Schreiber, N. M., Shapley, A. E., Erb, D. K., et al. 2011, ApJ, 731, 65 Gao, Y., \& Solomon, P. M. 2004, ApJ, 606, 271

Genzel, R., Tacconi, L. J., Gracia-Carpio, J., et al. 2010, MNRAS, 407, 2091 Giavalisco, M., Ferguson, H. C., Koekemoer, A. M., et al. 2004, ApJ, 600, L93 Graciá-Carpio, J., Sturm, E., Hailey-Dunsheath, S., et al. 2011, ApJ, 728, L7

Grazian, A., Fontana, A., de Santis, C., et al. 2006, A\&A, 449, 951

Helou, G., Malhotra, S., Hollenbach, D. J., Dale, D. A., \& Contursi, A. 2001, ApJ, 548, L73

Hwang, H. S., Elbaz, D., Magdis, G., et al. 2010, MNRAS, 409, 75

Karim, A., Schinnerer, E., Martínez-Sansigre, A., et al. 2011, ApJ, 730, 61

Kelson, D. D., \& Holden, B. P. 2010, ApJ, 713, L28

Kennicutt, R. C., Jr. 1989, ApJ, 344, 685

Kennicutt, R. C., Jr. 1998a, ARA\&A, 36, 189

Kennicutt, R. C., Jr. 1998b, ApJ, 498, 541
Kennicutt, R. C., Jr., Calzetti, D., Walter, F., et al. 2007, ApJ, 671, 333

Kriek, M., van Dokkum, P. G., Franx, M., Illingworth, G. D., \& Magee, D. K. 2009, ApJ, 705, L71

Laurent, O., Mirabel, I. F., Charmandaris, V., et al. 2000, A\&A, 359, 887

Leroy, A. K., Walter, F., Brinks, E., et al. 2008, AJ, 136, 2782

Luhman, M. L., Satyapal, S., Fischer, J., et al. 2003, ApJ, 594, 758

Luo, B., Bauer, F. E., Brandt, W. N., et al. 2008, ApJS, 179, 19

Lutz, D., Maiolino, R., Spoon, H. W. W., \& Moorwood, A. F. M. 2004, A\&A, 418,465

Lutz, D., Poglitsch, A., Altieri, B., et al. 2011, A\&A, 532, A90

Lutz, D., Valiante, E., Sturm, E., et al. 2005, ApJ, 625, L83

Magdis, G. E., Elbaz, D., Dickinson, M., et al. 2011, A\&A, 534, 15

Magnelli, B., Elbaz, D., Chary, R. R., et al. 2009, A\&A, 496, 57

Maiolino, R., Caselli, P., Nagao, T., et al. 2009, A\&A, 500, L1

Maiolino, R., Shemmer, O., Imanishi, M., et al. 2007, A\&A, 468, 979

Malhotra, S., Helou, G., Stacey, G., et al. 1997, ApJ, 491, L27

Malhotra, S., Kaufman, M. J., Hollenbach, D., et al. 2001, ApJ, 561, 766

Martin, D. C., Fanson, J., Schiminovich, D., et al. 2005, ApJ, 619, L1

Menéndez-Delmestre, K., Blain, A. W., Alexander, D. M., et al. 2007, ApJ, 655, L65

Murphy, E. J., Chary, R., Alexander, D. M., et al. 2009, ApJ, 698, 1380

Murphy, E. J., Chary, R.-R., Dickinson, M., et al. 2011, ApJ, 732, 126

Muzzin, A., van Dokkum, P., Kriek, M., et al. 2010, ApJ, 725, 742

Netzer, H., \& Trakhtenbrot, B. 2007, ApJ, 654, 754

Noeske, K. G., Weiner, B. J., Faber, S. M., et al. 2007, ApJ, 660, L43

Nordon, R., Lutz, D., Shao, L., et al. 2010, A\&A, 518, L24

Pannella, M., Carilli, C. L., Daddi, E., et al. 2009, ApJ, 698, L116

Papovich, C., Rudnick, G., Le Floc'h, E., et al. 2007, ApJ, 668, 45

Peng, Y., Lilly, S. J., Kovač, K., et al. 2010, ApJ, 721, 193

Pilbratt, G. L., Riedinger, J. R., Passvogel, T., et al. 2010, A\&A, 518, L1

Poglitsch, A., Waelkens, C., Geis, N., et al. 2010, A\&A, 518, L2

Pope, A., Chary, R.-R., Alexander, D. M., et al. 2008, ApJ, 675, 1171

Pope, A., Scott, D., Dickinson, M., et al. 2006, MNRAS, 370, 1185

Ranalli, P., Comastri, A., \& Setti, G. 2003, A\&A, 399, 39

Rex, M., Rawle, T. D., Egami, E., et al. 2010, A\&A, 518, L13

Rigby, J. R., Marcillac, D., Egami, E., et al. 2008, ApJ, 675, 262

Rodighiero, G., Cimatti, A., Gruppioni, C., et al. 2010, A\&A, 518, L25

Rujopakarn, W., Rieke, G. H., Eisenstein, D. J., \& Juneau, S. 2011a, ApJ, 726, 93

Rujopakarn, W., Rieke, G. H., Weiner, B. J., et al. 2011b, arXiv:1107.2921

Saintonge, A., Kauffmann, G., Kramer, C., et al. 2011a, MNRAS, 415, 32

Saintonge, A., Kauffmann, G., Wang, J., et al. 2011b, MNRAS, 415, 61

Sajina, A., Yan, L., Armus, L., et al. 2007, ApJ, 664, 713

Sales, D. A., Pastoriza, M. G., \& Riffel, R. 2010, ApJ, 725, 605

Salim, S., Rich, R. M., Charlot, S., et al. 2007, ApJS, 173, 267

Sanders, D. B., \& Mirabel, I. F. 1996, ARA\&A, 34, 749

Santini, P., Fontana, A., Grazian, A., et al. 2009, A\&A, 504, 751

Schmidt, M. 1959, ApJ, 129, 243

Shao, L., Lutz, D., Nordon, R., et al. 2010, A\&A, 518, L26

Shapiro, K. L., Genzel, R., Förster Schreiber, N. M., et al. 2008, ApJ, 682, 231

Siana, B., Smail, I., Swinbank, A. M., et al. 2009, ApJ, 698, 1273

Siana, B., Teplitz, H. I., Chary, R.-R., Colbert, J., \& Frayer, D. T. 2008, ApJ, 689,59

Solomon, P. M., Downes, D., Radford, S. J. E., \& Barrett, J. W. 1997, ApJ, 478, 144

Spoon, H. W. W., Koornneef, J., Moorwood, A. F. M., Lutz, D., \& Tielens, A. G. G. M. 2000, A\&A, 357,898

Tacconi, L. J., Genzel, R., Neri, R., et al. 2010, Nature, 463, 781

Teplitz, H. I., Chary, R., Elbaz, D., et al. 2011, AJ, 141, 1

Valiante, E., Lutz, D., Sturm, E., et al. 2007, ApJ, 660, 1060

Veilleux, S., Rupke, D. S. N., Kim, D., et al. 2009, ApJS, 182, 628

Wang, W., Cowie, L. L., Barger, A. J., Keenan, R. C., \& Ting, H. 2010, ApJS, 187,251

Weedman, D. W., Le Floc'h, E., Higdon, S. J. U., Higdon, J. L., \& Houck, J. R. 2006, ApJ, 638, 613

Wuyts, S., Förster Schreiber, N. M., Lutz, D., et al. 2011a, ApJ, 738, 106

Wuyts, S., Förster Schreiber, N. M., van der Wel, A., et al. 2011b, ApJ, 742, 96

Wuyts, S., Labbé, I., Schreiber, N. M. F., et al. 2008, ApJ, 682, 985 